\title{
THE SUMMER MEETING IN NEW HAVEN
}

The fifty-third Summer Meeting of the Society was held at Yale University, New Haven, Connecticut, Tuesday through Friday, September 2-5, 1947. The Mathematical Asscciation of America met on September 1-2 and The Institute of Mathematical Statistics on September 2-4. Over seven hundred people attended these meetings among whom were the following four hundred forty-three members of the Society:

J. C. Abbott, C. R. Adams, R. B. Adams, R. P. Agnew, E. J. Akutowicz, E. S. Allen, C. B. Allendoerfer, Warren Ambrose, R. C. Archibald, R. F. Arens, H. A. Arnold, H. E. Arnold, L. A. Aroian, M. G. Arsove, R. N. Ascher, H. T. R. Aude, Silvio Aurora, M. C. Ayer, Frank Ayres, W. L. Ayres, H. M. Bacon, F. E. Baker, D. H. Ballou, Joshua Barlaz, J. L. Barnes, H. P. Beard, F. S. Beckman. E. G. Begle, P. O. Bell, A. A. Bennett, Stefan Bergman, Felix Bernstein, A. H. Black, Garrett Birkhoff, D. H. Blackwell, R. P. Boas, H. W. Bode, J. W. Bower, J. G. Bowker, H. R. Brahana, A. T. Brauer, Richard Brauer, J. V. Breakwell, Robert Breusch, J. R. Britton, Foster Brooks, Bailey Brown, R. H. Brown, E. F. Buck, R. C. Buck, L. H. Bunyan, M. L. Burke, F. J. H. Burkett, W. K. Burroughs, Hobart Bushey, J. H. Bushey, W. H. Bussey, Albert Cahn, S. S. Cairns, B. H. Camp, G. C. Campbell, R. E. Carr, W. B. Carver, G. F. Carrier, C. R. Cassity, W. B. Caton, Omar Catunda, K. Chandrasekharan, Harold Chatland, W. F. Cheney, W. L. Chow, D. E. Christie, K. L. Chung, C. E. Clark, H. E. Clarkson, A. B. Coble, I. S. Cohen, L. W. Cohen, Nancy Cole, R. H. Cole, L. A. Colquitt, Esther Comegys, Erben Cook, T. F. Cope, L. P. Copeland, W. H. H. Cowles, V. F. Cowling, M. J. Cox, H. S. M. Coxeter, C. C. Craig, Jane Cronin, L. L. Cronvich, C. W. Crouse, E. L. Crow, H. B. Curry, G. B. Dantzig, M. D. Darkow, F. H. Davidson, D. R. Davis, M. D. Davis, F. F. Decker, C. E. Dimick, Bernard Dimsdale, L. L. Dines, M. P. Dolciani, M. D. Donsker, H. L. Dorwart, Y. N. Dowker, Arnold Dresden, Nelson Dunford, W. L. Duren, William H. Durfee, Ben Dushnik, Beno Eckmann, Samuel Eilenberg, Churchill Eisenhart, F. M. Ellis, Murray Ellis, Bernard Epstein, Paul Erdös, M. W. Eudey, H. P. Evans, B. G. Farley, Herbert Federer, J. M. Feld, William Feller, A. D. Fialkow, F. A. Ficken, N. J. Fine, H. A. Fisher, W. B. Fite, W. W. Flexner, M. M. Flood, L. R. Ford, R. M. Foster, F. H. Fowler, A. H. Fox, R. H. Fox, J. S. Frame, Orrin Frink, R. E. Fullerton, H. L. Garabedian, L. J. Gårding, C. S. Gardner, R. S. Gardner, H. M. Gehman, Hilda Geiringer, B. H. Gere, Irving Gerst, B. P. Gill, R. E. Gilman, Wallace Givens, A. M. Gleason, H. E. Goheen, Michael Goldberg, R. O. Goodman, M. J. Gottlieb, M. C. Graustein, H. J. Greenberg, Leonard Greenstone, Edison Greer, V. G. Grove, Theodore Hailperin, D. W. Hall, Marshall Hall, P. R. Halmos, O. H. Hamilton, R. W. Hamming, G. H. Handelman, B. I. Hart, K. E. Hazard, G. A. Hedlund, A. E. Heins, M. H. Heins, R. G. Helsel, Aaron Herschfeld, Fritz Herzog, Edwin Hewitt, T. H. Hildebrandt, Einar Hille, I. I. Hirschman, G. P. Hochschild, Banesh Hoffmann, T. R. Hollcroft, R. H. Hoskins, Harold Hotelling, A. S. Householder, C. C. Hsiung, H. T. Hsu, E. M. Hull, Witold Hurewicz, W. A. Hurwitz, R. E. Huston, L. C. Hutchinson, S. B. Jackson, Nathan Jacobson, J. J. Janko, Herbert Jehle, E. D. Jenkins, S. A. Jennings, R. N. Johanson, R. B. Johnson, R. F. Johnson, C. W. Jordan, Aida Kalish, Irving Kaplansky, William Karush, M. E. Kellar, L. M. Kelly, A. J. Kempner, D. E. Kibbey, Fred Kiokemeister, 
W. J. Klimczak, E. R. Kolchin, H. S. Konijn, C. F. Kossak, M. Z. Krzywoblocki, Jack Laderman, W. D. Lambert, H. G. Landau, R. E. Langer, E. H. Larguier, J. P. LaSalle, H. L. Lee, J. R. Lee, E. L. Lehmann, Marguerite Lehr, R. A. Leibler, Walter Leighton, F. C. Leone, Howard Levene, W. J. LeVeque, Norman Levinson, D. C. Lewis, F. A. Lewis, M. A. Lipschutz, B. J. Lockhart, Z. L. Loflin, A. J. Lohwater, W. R. Longley, Lee Lorch, C. I. Lubin, V. O. McBrien, E. D. McCarthy, R. B. McClenon, Dorothy McCoy, N. H. McCoy, E. A. McDougle, W. H. McEwen, L. A. MacColl, G. W. Mackey, G. R. MacLane, H. F. MacNeish, H. B. Mann, Morris Marden, M. H. Martin, W. T. Martin, P. R. Masani, F. J. Massey, F. I. Mautner, A. E. Meder, G. M. Merriman, E. L. Mickelson, E. J. Miles, A. N. Milgram, Knox Millsaps, S. Minakshisundaram, H. J. Miser, J. M. Mitchell, E. B. Mode, Deane Montgomery, J. C. Montgomery, F. V. E. Morfoot, C. N. Moore, T. W. Moore, E. M. Morenus, R. K. Morley, D. S. Morse, Frederick Mosteller, H. T. Muhly, C. G. Mumford, W. R. Murray, J. R. Musselman, A. L. Nelson, P. F. Neményi, E. D. Nering, O. E. Neugebauer, John von Neumann, C. V. Newsom, E. N. Nilson, G. E. Noether, P. B. Norman, E. P. Northrop, H. W. Norton, Lawrence Norwood, R. E. O'Connor, P. S. Olmstead, Paul Olum, T. G. Ostrom, E. R. Ott, M. H. Payne, D. K. Pease, W. H. Pell, P. M. Pepper, R. I. Pepper, Sam Perlis, H. R. Phalen, George Piranian, J. G. Pocock, Harry Pollard, C. R. Polley, George Pólya, William Prager, G. B. Price, A. L. Putnam, G. Y. Rainich, J. F. Randolph, Anatol Rapoport, R. B. Rasmusen, C. H. Rawlins, G. E. Raynor, L. J. Reed, C. J. Rees, Mina Rees, W. T. Reid, Irving Reiner, B. P. Reinsch, Helene Reschovsky, C. N. Reynolds, R. G. D. Richardson, C. E. Rickart, P. R. Rider, F. D. Rigby, John Riordan, J. F. Ritt, E. K. Ritter, H. E. Robbins, H. M. Roberts, M. S. Robertson, L. B. Robinson, L. V. Robinson, Saul Rosen, J. B. Rosser, M. F. Rosskopf, E. H. Rothe, Herman Rubin, Charles Saltzer, H. E. Salzer, Hans Samelson, Arthur Sard, Max Sasuly, F. E. Satterthwaite, A. T. Schafer, R. D. Schafer, M. M. Schiffer, A. E. Schild, O. F. G. Schilling, R. W. Schmied, I. J. Schoenberg, K. C. Schraut, J. E. Schubert, Abraham Schwartz, G. E. Schweigert, W. T. Scott, C. E. Sealander, C. H. W. Sedgewick, I. E. Segal, A. S. Shapiro, H. N. Shapiro, H. S. Sharp, L. W. Sheridan, Seymour Sherman, Max Shiffman, Marlow Sholander, D. T. Sigley, Milton da Silva Rodrigues, D. N. Silver, M. M. Slotnick, D. M. Smiley, M. F. Smiley, C. V. L. Smith, F. C. Smith, P. A. Smith, R. E. Smith, W. M. Smith, Ernst Snapper, W. S. Snyder, Andrew Sobczyk, Herbert Solomon, E. H. Spanier, A. H. Sprague. C. E. Springer, D. E. Spencer, V. E. Spencer, M. E. Stark, E. P. Starke, Rothwell Stephens, F. M. Stewart, R. W. Stokes, R. R. Stoll, R. C. Strodt, Walter Strodt, M. C. Suffa, A. C. Sugar, M. M. Sullivan, J. L. Synge, Gabor Szego, A. H. Taub, J. S. Taylor, Feodor Theilheimer, J. E. Thompson, D. L. Thomsen, R. M. Thrall, W. J. Thron, H. S. Thurston, Gerhard Tintner, Leonard Tornheim, M. M. Torrey, J. I. Tracey, W. J. Trjitzinsky, C. A. Truesdell, Y. W. Tschen, Bryant Tuckerman, J. W. Tukey, H. S. Uhler, S. M. Ulam, E. P. Vance, H. E. Vansant, Philon Vassiliou, D. F. Votaw, Abraham Wald, H. M. Walker, R. J. Walker, Henry Wallman, J. L. Walsh, R. M. Walter, J. B. Walton, C. W. Watkeys, G. C. Webber, Alexander Weinstein, B. A. Welch, D. W. Western, G. W. Whitehead, A. L. Whiteman, P. M. Whitman, Hassler Whitney, D. V. Widder, L. R. Wilcox, S. S. Wilks, M. F. Willerding, John Williamson, G. M. Wing, R. M. Winger, H. A. Wood, Jacob Wolfowitz, F. M. Wright, Bertram Yood, J. W. T. Youngs, J. A. Zilber, Oscar Zariski.

On Tuesday afternoon, Wẻdnesday, Thursday and Friday mornings, Professor Oscar Zariski of Harvard University gave the Col- 
loquium Lectures on Abstract algebraic geometry. President Einar Hille, Professor A. B. Coble, Professor T. H. Hildebrandt, and Vice President L. R. Ford presided in turn at these lectures.

On Thursday afternoon Professor S. S. Wilks of Princeton University gave an address on Sampling theory of order statistics. Professor Harold Hotelling presided.

Presiding officers for the sessions of contributed papers were: Applied Mathematics, Tuesday afternoon, Professor William Prager; Geometry, Tuesday afternoon, Reverend R. E. O'Connor; Analysis, Wednesday morning, Professor W. T. Reid; Algebra, Wednesday morning, Professor P. M. Whitman; Applied Mathematics, Thursday morning, Professor W. T. Martin; Topology, Thursday morning, Professor G. E. Schweigert; Statistics and Probability, Thursday afternoon, Professor J. W. Tukey; Analysis, Thursday afternoon, Professor Gabor Szegö; Analysis, Friday morning, Professor G. B. Price; Algebra, Friday morning, Professor M. F. Smiley.

There was a business meeting Wednesday morning at which President Einar Hille presided.

The general sessions were all held in the auditorium of Sprague Hall and the sections were held in this room and in 201 Harkness Hall.

Registration headquarters were in Branford College. Rooms in Branford, Jonathan Edwards, and Saybrook Colleges were available to those attending the meetings. Meals were served in the Cafeteria of the University Dining Hall. On Wednesday and Thursday mornings three tours to different parts of the campus were conducted simultaneously. One included the Sterling Memorial Library and the Payne Whitney Gymnasium; another the Gallery of Fine Arts, and the third the Peabody Museum. The picnic and swimming at Hammonasset State Park on Wednesday afternoon were enjoyed by over four hundred people. Wednesday noon there was a trip to visit the insurance companies in Hartford, Connecticut. The mathematicians were the guests of the Connecticut Mutual Life Insurance Company at a luncheon served in the Company's cafeteria. On Wednesday just before the picnic there was a tour to the Connecticut Agricultural Experiment Station. Throughout the entire week there was an exhibition of early books outstanding in the history of mathematics.

On Tuesday afternoon, the ladies of the mathematics faculty served tea in the President's Room, Woolsey Hall.

On Tuesday evening, an organ recital was given by Professor $\mathrm{H}$. Frank Bozyan of Yale University.

A group photograph of those attending the meeting was taken on Wednesday at 1:30 P.M. 
The dinner for the three organizations was served in the University Dining Hall. Professor W. R. Longley was toastmaster. Director E. W. Sinnott of the Sheffield Scientific School welcomed the mathematicians and their guests. President Einar Hille of the Society, President L. R. Ford of the Association and President William Feller of the Institute talked about the rapid growth of the three organizations in membership and activities. Professor P. R. Rider proposed a resolution, unanimously approved, expressing the appreciation of the members of the three organizations to the administration of Yale University, the local committee and all who contributed to the success of the meetings.

The Board of Trustees of the Society met Wednesday at 2:00 P.M.

The Council met in the lounge of Jonathan Edwards College at 8:00 P.M. on Tuesday.

The Secretary announced the election of the following eighty-eight persons to ordinary membership in the Society:

Professor José Abdelhay, University of Brazil;

Mr. William Attix Allen, Naval Research Laboratory, Washington, D. C.;

Mr. Alfred Titus Anderson, Sewanhaka High School, Floral Park, N.Y., and Cooper Union, New York, N. Y.;

Mr. John Jacob Andrews, St. Louis University;

Dr. Franz L. Alt, Ballistic Research Laboratories, Aberdeen Proving Ground, Md.;

Mr. Frank Arena, University of Michigan;

Mr. Eugene August Behmer, Minnesota State Highway Department, St. Paul, Minn.;

Mr. Paul Walter Berg, New York, N. Y.;

Dr. Felix Adalbert Behrend, University of Melbourne;

Professor Enrique Loizelier Blanco, University of Madrid;

Mr. Henry David Block, Iowa State College of Agriculture and Mechanical Arts;

Mr. Darrell Hastings Browne, Buffalo, N. Y.;

Mr. Elmer Jesse Bryson, Shell Development Company, Emeryville, Calif.;

Mr. Carlos Alberto Aragão de Carvalho, Federal District, Brazil;

Mr. Charles William Cassel, Jr., University of Dayton;

Professor Benedito Castrucci, University of São Paulo;

Mr. Leo George Chelius, Carbide and Carbon Chemicals Corporation, Oak Ridge, Tenn.;

Mr. Peter Joseph Cocuzza, Brooklyn, N. Y.;

Mr. Robert Reginald Coveyou, Monsanto Chemical Company, Knoxville, Tenn.;

Mr. Charles William Crouse, Manufacturers Casualty Insurance Company, Philadelphia, Pa.;

Miss Nancy Lee Davidson, U. S. Naval Ordnance Test Station, Inyokern, Calif.;

Mr. David Bliss Dekker, University of California;

Mr. Lars Niclas Enequist, Bendix Aviation Corporation, Teterboro, N. J.;

Mr. Joseph Epstein, Philosophy Department, Columbia University;

Mr. Robert M. Exner, Syracuse University;

Mr. Edison Farah, University of São Paulo;

Mr. Thorleif Moland Fostvedt, University of Alberta;

Mr. Peter Alden Franken, New York, N. Y.; 
Professor Fernando Furquim de Almeida, University of São Paulo;

Mr. Landis Gephart, University of Dayton;

Mr. Alvercio Moreira Gomes, University of Brazil;

Miss Elza Furtado Gomide, University of São Paulo;

Mr. Harry Herbert Goode, Office of Naval Research, Sands Point, N. Y.;

Mr. Earl Vincent Greer, Bethany-Peniel College, Bethany, Okla.;

Mr. Ralph Hafner, University of Dayton;

Mr. Norman Shannon Hall, Jr., University of California;

Mr. Warren Maurice Hirsch, New York University;

Mr. Temple Rice Hollcroft, Jr., N. C. A., Washington, D. C.;

Mr. Morton Allan Hyman, Naval Ordnance Laboratory, Washington, D. C.;

Mr. Eri T. Jabotinsky, Jerusalem, Palestine;

Mr. Reginald Charles Jacka, University of Alberta;

Professor Jaroslav Joseph Janko, University of Technical Sciences of Prague;

Mr. Robert Kahal, Polytechnic Institute of Brooklyn;

Mr. Herman Kahn, Los Angeles, Calif.;

Professor Joseph Kampé de Fériet, University of Lille;

Mr. Edward Lynn Kaplan, Naval Ordnance Laboratory, Washington, D. C.;

Professor Hendrik Douwe Kloosterman, University of Leiden;

Mr. Simon Harold Lachenbruch, National Bureau of Standards, Washington, D. C.;

Mr. Mario Laserna, New York, N. Y.;

Dean Ta Chung-Heng Li, National Fuh Tan University, Shanghai, China;

Professor Candido Lima a Silva Dias, University of São Paulo;

Miss Miriam Amalie Lipschutz, Massachusetts Institute of Technology;

Mr. Sherman Cabot Lowell, New York University;

Mr. Harold Edwin Malde, Portland, Ore.;

Dr. J. Carson Mark, Los Alamos Scientific Laboratories, Los Alamos, N. Mex.;

Mr. David G. Mead, West Orange, N. J.;

Mr. Sidney Melmore, Acomb, York, England;

Mr. Hasan Hasan Mostafa, Syracuse University;

Professor Antonio Aniceto Monteiro, University of Brazil;

Mr. Luiz Henrique Jacy Monteiro, University of São Paulo;

Mr. Wolfe Mostow, Bureau of Ships, Navy Department, Washington, D. C.;

Professor Leopoldo Nachbin, University of Brazil;

Mr. K. R. Narayana Row, Carnegie Institute of Technology;

Professor Ernesto Luis Oliveira, Jr., University of Brazil;

Professor Francisco Mendes de Oliveira Castro, School of Engineering, University of Brazil;

Professor Theodore Gleason Ostrom, Montana State University;

Dr. Mary Hewlett Payne (Mrs. W. T.), University of Detroit;

Professor Marillia Chaves Peixoto, School of Engineering, University of Brazil;

Professor Maurico Matos Peixoto, School of Engineering, University of Brazil;

Dr. Charles Dillon Perrine, Córdoba, Argentina;

Mr. Robert Richard Putz, Extension Division, University of California;

Professor Cortez Benjamin Rader, University of Houston;

Mr. Otho Mills Rasmussen, University of Kansas;

Professor C. Carl Robusto, St. John's University;

Miss Sally Irene Rothstein, University of Wisconsin;

Dr. John Paul Scholz, Bartol Research Foundation, Franklin Institute, Swarthmore, Pa.

Mr. Benjamin L. Schwartz, Carnegie Institute of Technology; 
Professor Raymond Coffey Staley, University of North Dakota;

Miss Irene Anne Stegun, Mathematical Tables Project, National Bureau of Standards, New York, N. Y.;

Professor Haldum Koca Taskin, Milwaukee School of Engineering, Milwaukee, Wis.;

Mr. Gino Turrin, Buenos Aires, Argentina;

Professor Paul Vincensini, University of Besançon;

Mr. John Howell White, U. S. Naval Academy;

Dr. Margaret Frances Willerding, Washington University;

Professor Martin Wright, University of Houston;

Mr. Norman Joseph Zabb, Brooklyn, N. Y.;

Mr. Lotfi Asker Zadeh, Electrical Engineering Department, Columbia University;

Mr. Julian Joel Zeig, College of the City of New York.

It was reported that the following had been elected to membership on nomination of institutional members as indicated:

Harvard University: Dean Gordon Maskew Fair;

Institute for Advanced Study: Professor Wei Liang Chow and Mr. Sven Herbert Hilding.

The Secretary announced that the following had been admitted to the Society in accordance with reciprocity agreements with various mathematical organizations: Deutsche Mathematiker Vereinigung: Professor Paul Funk, Technische Hochschule, Vienna, Austria; Dr. Maximilian Joseph Pinl, University of Cologne; Professor Hans Zassenhaus, University of Hamburg. London Mathematical Society: Professor Harold Davenport, University of London; Dr. Arthur Erdélyi, Edinburgh University; Dr. Hans Arnold Heilbronn, University of Bristol; Dr. Archibald James Macintyre, University of Aberdeen; Dr. Dan Pedoe, Westfield College, London; Professor Harry Raymond Pitt, Queen's University, Belfast, Northern Ireland; Professor Harold Stanley Ruse, University of Leeds; Professor Arthur Geoffrey Walker, University of Sheffield. Swiss Mathematical Society: Professor Johannes Gualtherus van der Corput, University of Amsterdam; Professor Walter Saxer, Eidgenössische Technische Hochschule, Zurich; Professor Albert Pfluger, Eidgenössische Technische Hochschule, Zurich; Professor Eugenio G. Togliatti, University of Genoa; Professor Georges Valiron, University of Paris. Unione Matematica Italiana: Professor Carlo Miranda, University of Naples.

The Council had adopted by mail vote the report of the Policy Committee for Mathematics, favoring the meeting in connection with the Mexico City meeting of UNESCO for a preliminary discussion of the re-establishment of an International Mathematical Union. Subsequent to this report of the Policy Committee and in view of the unwillingness of the International Council of Scientific Unions to sponsor such a meeting in Mexico City, it was agreed by the Policy 
Committee to abandon the meeting that had been contemplated for Mexico City.

The Council had also approved by mail vote the publication of the Proceedings of the Symposium on Non-linear problems in mechanics of continua, held at Brown University on August 2-4, 1947.

The following appointments by President Einar Hille were reported: Professor Nelson Dunford as representative of the Society at the inauguration of Rosemary Park as President of Connecticut College on May 17, 1947; Professor Morris Marden as representative of the Society at the inauguration of Nelson Vance Russell as President of Carroll College on May 17, 1947; Dr. G. E. Forsythe as representative of the Society at the inauguration of Raymond Bernard Allen as President of the University of Washington on May 2224, 1947 ; Professor H. S. MacDougal as representative of the Society at the inauguration of Fred H. Leinbach as President of South Dakota State College on June 2, 1947; Professor J. W. Tukey as representative of the Society on the Joint Committee for the Development of Statistical Applications in Engineering and Manufacturing; Professors P. R. Rider (Chairman), R. H. Bruck, J. E. Case, T. L. Downs, Walter Leighton, Messrs. C. W. Mathews, Marlow Sholander, Professor W. H. Roever as a Committee on Arrangements for the November, 1947, meeting at Washington University; Professors T. H. Hildebrandt (Chairman), Arnold Dresden, J. R. Kline, Marston Morse, P. A. Smith, Dr. Warren Weaver as a committee to study the problems of the library and housing (joint committee of Council and Trustees); Professors Eric Reissner (three years), J. J. Stoker (two years), William Prager (one year) as an Editorial Committee for Applied Mathematics Symposium Proceedings, with Professor Reissner appointed Chairman of the Committee as now constituted.

The Naval Ordnance Laboratory has appointed Dr. C. A. Truesdell as a member of the Laboratory's Committee of Professional Society Representatives for the specific purpose of liaison with the American Mathematical Society.

It was reported that Professor Hermann Weyl had accepted the invitation to deliver the Josiah Willard Gibbs Lecture at the 1948 Annual Meeting.

The following dates of meetings in 1948 were approved: February 28 in New York City; February 28 in Chicago, Illinois; April 16-17 in New York City; April 16-17 in Ann Arbor, Michigan; April 17 in Berkeley, California. The Council also voted in favor of holding a meeting in Vancouver, British Columbia in June, 1948, the details 
to be left to the Associate Secretary for the far west.

On recommendation of the Colloquium Editorial Committee, the Council voted to accept for publication in the Colloquium Series the manuscript of Professor R. L. Wilder, entitled Topology of manifolds. It was further voted to invite Professor G. A. Hedlund of the University of Virginia to deliver a series of Colloquium Lectures at the 1949 Summer Meeting.

On recommendation of the Mathematical Surveys Editorial Committee, the Council voted to accept for publication in that series a manuscript entitled Valuation theory by Professor O. F. G. Schilling and one entitled Geometry of the zeros of a polynomial by Professor Morris Marden.

The Finance Committee for the Birkhoff Memorial Project reported that, as of August 18, 1947, a total of $\$ 6,616.20$ had been received in subscriptions and contributions as a result of the preliminary solicitation among friends of Professor Birkhoff and members of the Society. The Council and Trustees had recommended at an earlier date that the project proceed as soon as the sum of $\$ 6,000$ had been received. Hence the Editorial Committee for the project has now been requested to begin the task of preparing the material for publication. The Council and Trustees approved the tentative price of $\$ 18.00$ per set and the special pre-publication discount of 30 percent to members of the Society. It was further agreed by these two groups that the final price of the volumes should not be set before the contract for their printing has been let and that no subscriptions at the pre-publication rate should be accepted after December 31, 1947.

It was agreed to change the Society's Committee on Publicity to a joint Committee on Publicity of the Society and the Association. This action had been concurred in by the Board of Governors of the Association. Representatives of other organizations which may meet from time to time with the Society and the Association will be added to the committee as the occasion arises.

On recommendation of the Committee on Applied Mathematics, President Hille was authorized to appoint a Committee on Known Results. This committee is to make a survey of available compendia, including tables of infinite series, solutions of differential equations, et cetera, but not numerical tables; it is also hoped that this committee will propose possible action by the Society to remedy deficiencies revealed by such a survey.

Professor Szolem Mandelbrojt has been invited to deliver an address at the November, 1947, meeting at Washington University. 
Professors R. P. Agnew and W. M. Whyburn were appointed representatives of the Society on the Council of the American Association for the Advancement of Science for the year 1948.

Professor T. H. Hildebrandt and Dean M. H. Ingraham were nominated as representatives of the Society in the Division of Physical Sciences of the National Research Council for the three-year period beginning July 1,1948 .

It was announced that the Secretary had submitted a report to the Rockefeller Foundation in July, 1947, regarding the activities of the Policy Committee for Mathematics. The Council and Trustees voted to appropriate a sum of $\$ 500$ per year for the work of the Policy Committee which has been financed in the past by generous grants from the Rockefeller Foundation.

The Committee on Aid to Devastated Libraries reported that the first shipment of books and journals was on its way to twelve libraries in ten different countries. In response to the appeal published in the spring, a number of cash contributions, amounting in total to somewhat over $\$ 300$, had been received. Among these contributions there are several from student bodies in different institutions. A gift of $\$ 250$ was made by the Mathematical Association of America at the New Haven meeting. Further gifts of books and of periodicals were received from a number of individuals as well as from a number of mathematical journals.

The Council recommended to the Trustees that the Editors of Mathematical Reviews be authorized to publish in alternate years a complete list of the journals from which abstracts are made.

The President was authorized to appoint a committee to study the present organization of the Society and to make recommendations at an early date for such changes as they deem to be necessary to adjust the organization of the Society to present conditions.

In the Council Meeting and later at the Business Meeting of the Society it was announced that Mrs. Genevra Barrett Hutchinson, who died recently, had made a bequest of one thousand dollars to the Society in memory of her late husband, John Irwin Hutchinson, and in accordance with his wishes. John Irwin Hutchinson (1867-1935) was successively instructor, associate professor, and professor of mathematics at Cornell University from 1894 to 1935 . He was one of the first cooperating editors of the Transactions of the Society, serving from 1902 to 1915 . In 1910 he was vice president of the Society. He made frequent contributions of research papers, chiefly in analysis, but also in algebra and geometry. Two of his achievements deserve particular mention for their permanent value and the atten- 
tion they attracted here and abroad: the introduction of the isometric circle in connection with automorphic functions, and the discovery of the infinite group of birational transformations of the general Kummer surface.

Abstracts of most of the papers read by title were published in the September issue of this Bulletin. Abstracts of the rest of the papers read by title and all of the papers read in person follow below. Abstracts whose numbers are followed by the letter " $t$ " represent papers presented by title. Mr. Graves was introduced by Professor R. H. Cameron, Mr. Hahn by Professor J. H. Roberts, Mr. Reynolds by Professor A. T. Brauer, and Mr. Wall by Dr. H. W. E. Schwerdtfeger. The following papers were read by: 366, Professor Perlis; 367, Professor Hall; 378, Dr. Ayer; 381, Dr. Piranian; 386, Professor Helsel; 403, Dr. Ulam; 410, Professor Synge; 411, Dr. Neményi; 412, Mr. Prim; 414, Dr. Mary H. Payne; 420, Mr. Schwartz; 430, Dr. Aroian; 434, Dr. Lehmann; 438, Professor Fox; 439, Professor Samelson.

\section{Algebra ANd Theory of Numbers}

362. A. T. Brauer: On the irreducibility of polynomials with large third coefficient.

Let $f(x)=x^{n}+a^{n-1}+\cdots+a_{n}$ be a polynomial with integral rational coefficients. It was proved by Perron (J. Reine Angew. Math. vol. 132 (1907) pp. 288-307) that $f(x)$ is irreducible in the field of rational numbers if $a_{n} \neq 0$ and $a_{2}>16^{n-1}\left(\left|a_{1}\right|+\left|a_{3}\right|\right.$ $\left.+\cdots+\left|a_{n}\right|\right)^{2}$. Lipka (Math. Ann. vol. 118 (1941-1943) pp. 235-245) proved that $16^{n-1}$ can be replaced by $9 / 2$. In this paper this result is improved further. (Received July $28,1947$.

363. Richard Brauer: On the Schur index of the representations of groups of finite order. Preliminary report.

It is shown that the Schur index of a representation $F$ of a group $G$ of finite order with regard to a field $K$ of characteristic 0 can be expressed as a product of Schur indices of representations of suitable subgroups $H$ of $G$. Each group $H$ contains a cyclic normal subgroup $A$ such that $H / A$ is a group of prime power order $p^{r}$; the order of $A$ is not divisible by $p$. The Schur index of the representation of $H$ is the exact contribution of $p$ to the Schur index of $F$. This shows in particular that the whole problem can be reduced to one dealing with soluble groups. (Received July $30,1947$.

364. Mary P. Dolciani: On the representation of integers by quadratic forms.

Let $S$ be a quadratic form $\sum_{i, j=1}^{n} a_{i j} x_{i} x_{j}$ in the variables $x_{1}, \cdots, x_{n}$ in which the $a_{i i}$ and $2 a_{i j}$ are rational integers; and let $m$, the rank of $S$, be greater than or equal to four. For a given integer $c$ the problem is to determine whether or not there exist integral solutions of the equation $S=c$ and to give a quantitative measure of the number of such solutions of $S=c$. Associating with $S$ a particular diagonal positive form $P$, the author considers the sum $A(\epsilon)=\sum \exp (-\pi \in P)$ extended over all 
integral solutions of $S=c$. By use of a generalization of a method employed by Siegel (Amer. J. Math. vol. 63 (1941) pp. 658-680), it is proved that if $S$ is an indefinite form which represents $c$ in the ring of $p$-adic integers for all primes $p$, then, as $\epsilon$ tends to zero through positive values, the expression $\epsilon^{(m / 2)-1} A(\epsilon)$ approaches a positive limit. Analogous results are also obtained for $S$ a definite form, this case having been already treated by Kloosterman (Acta Math. vol. 49 (1926) pp. 407-464) and by Ross and Pall (Amer. J. Math. vol. 68 (1946) pp. 47-65). (Received July 26, 1947.)

\section{Ben Dushnik: $A$ property of a set of permutations.}

Let $S$ be a set of $m$ elements. For any positive integer $k \leqq m$, let $T$ be a set of permutations of the elements of $S$ which possesses the following property: $(\alpha)$ For any $k$ elements of $S$, say $a_{1} a_{2}, \cdots, a_{k}$, and for $j \leqq k$ there exists a permutation in $T$ in which $a_{j}$ follows all the other elements $a_{i}$ with $i \neq j$. The smallest number of permutations which a set $T$ with property $(\alpha)$ must possess is denoted by $D(m, k)$. For a given $m$, it is difficult to obtain the value of $D(m, k)$ for small $k(>2)$; however, it is possible to obtain an explicit formula for $D(m, k)$ if $k$ is sufficiently large. (Received July $28,1947$.

\section{John Dyer-Bennet and Sam Perlis: On the center of a nilpo- tent algebra.}

Let $N$ be a nilpotent algebra with center $C$. Then $C$ is an ideal of $N$ if $N$ has index two or three, but an example is given in which $C$ is not an ideal, $N$ having order seven and index four, $N^{2}$ having order four, $N^{3}$ having order one. It is shown that $C$ always is an ideal if $N$ has order $n<7$, and, more generally, if $N$ has index four or five and the orders of certain difference algebras $N^{i}-N^{i+1}$ are suitably restricted. If $N$ has order $n$ and index $n+1$ (the maximum index for the given order), then $N$ is commutative, being generated by a single quantity. (Received July 25, 1947.)

367. Marshall Hall and Tibor Rado: On Schreier systems in free groups.

A system of elements in a free group is called a Schreier system if it contains the beginning sections of every element in the system. Proceeding in a way inverse to that followed by Schreier, it is shown that every Schreier system can be the coset representatives of a subgroup, and in fact a parametric representation is given for all subgroups with a fixed Schreier system of coset representatives. The word problem is solvable for subgroups given by this parametric representation. For a shortest Schreier system belonging to a subgroup, it is shown that the subgroup generators derived from the system automatically satisfy the Nielsen conditions for reduced generators. (Received May 19, 1947.)

\section{S. A. Jennings: The u-algebra of a nilpotent restricted Lie alge-} bra. Preliminary report.

Jacobson has shown that the Birkhoff-Witt algebra $u$ belonging to a nilpotent restricted Lie algebra $L$ over a field of characteristic $p$ is itself a nilpotent algebra (Trans. Amer. Math. Soc. vol. 50 (1941) pp. 15-25). In this note bases for the ideals $u^{w}, w=1,2, \cdots$, are determined in terms of the elements of a series of ideals of $L$ analogous to the dimensional subgroups $\bmod p$ of a $p$-group. These results are used to 
investigate the structure of certain Lie algebras associated with p-groups. Similar methods may be used to study the Birkhoff-Witt algebra of any nilpotent Lie algebra over an arbitrary field. (Received July 31, 1947.)

\section{Irving Kaplansky: Rings with a polynomial identity.}

M. Hall has observed that any division ring satisfying $(x y-y x)^{2} z=z(x y-y x)^{2}$ is a field or quaternion algebra. This is generalized as follows: if $D$ is a division ring for which there exists a non-commutative polynomial $f\left(x_{i}\right)$ such that $f=0$ for $x_{i}$ in $D$, then $D$ is finite-dimensional over its center $C$. If the degree of $f$ is less than $6,[D: C]$ is 1 or 4 . An essential step in the proof is the fact that the free non-commutative algebra generated by two indeterminates has a complete set of finite-dimensional representations. It is conversely true that any algebra of finite order satisfies a polynomial identity. (Received July 25, 1947.)

370. W. J. LeVeque: The order of magnitude of number-theoretic functions.

Let $f(p)$ be a strongly additive arithmetic function, with $|f(p)| \leqq 1$, let $\dot{A}_{n}=\sum_{p \leqq n} f(p) / p, B_{n}=\left(\sum_{p \leqq n} f^{2}(p) / p\right)^{1 / 2}$, where $p$ is a prime number, and let $\omega_{1}, \omega_{2}, \omega$ be real numbers. Then if $B_{n} \rightarrow \infty$ with $n$, the number of integers $m, 0<m \leqq n$, for which simultaneously $f(m)<A_{n}+\omega_{1} B_{n}, f(m+1)<A_{n}+\omega_{2} B_{n}$ is $n D\left(\omega_{1}\right) D\left(\omega_{2}\right)+o(n)$, where $D(\omega)$ is the Gaussian integral. In particular, if $\nu(m)$ denotes the number of distinct prime divisors of $m$, the number of positive integers $m \leqq n$ for which $\nu(m)<\log \log n+\omega_{1}(\log \log n)^{1 / 2}$ and $\nu(m+1)<\log \log n+\omega_{2}(\log \log n)^{1 / 2}$ is $n D\left(\omega_{1}\right) D\left(\omega_{2}\right)+o(n)$. It can be shown from this that the number of $m \leqq n$ for which $\nu(m)<\nu(m+1)+\omega(2 \log \log n)^{1 / 2}$ is $n D(\omega)+o(n)$, and hence that the number of $m \leqq n$ for which $d(m)<d(m+1) \cdot 2^{\omega}(2 \log \log n)^{1 / 2}$ is $n D(\omega)+o(n)$, if $d(m)$ is the number of divisors of $m$. A simpler proof is given for the special case of a theorem of Erdös and Kac (Amer. J. Math. vol. 62 (1940) pp. 738-742) where $f(m)=\nu(m)$. The proofs rely on Brun's method and the central limit theorem from the calculus of probability. The first theorem mentioned above was stated without proof by Erdös (Ann. of Math. vol. 47 (1946) p. 17). (Received July 23, 1947.)

\section{1t. D. C. Murdoch: A theorem on nilpotent groups.}

Corresponding to a fixed element $a$ of a group $G$ a series of subgroups $G_{a}^{(0)} \supseteq G_{a}^{(1)}$ $\supseteq G_{a}^{(2)} \supseteq \cdots$ may be defined where $G_{a}^{(0)}=G$ and $G_{a}^{(i)}$ is the subgroup generated by all commutators $g^{-1} a^{-1} g a$ where $g$ is an element of $G_{a}^{(i-1)}$. Each $G_{a}^{(i)}$ is a normal subgroup of $G_{a}^{(i-1)}$. If this series terminates in the unit element the element $a$ will be called a nilpotent element of $G$. If $G$ is finite it is shown that the nilpotence of every element of $G$ implies the nilpotence of $G$ in the usual sense. (Received July 30,1947.)

\section{Irving Reiner: A generalization of Meyer's theorem.}

The following theorem of A. Meyer (Journal für Mathematik vol. 103 (1888) p. 98 ) is generalized: Every properly primitive binary quadratic form represents infinitely many primes in any preassigned arithmetic progression $M x+N$ consistent with the generic character of the form, where $M$ and $N$ are relatively prime. The generalization consists of replacing the set of classes of properly primitive binary quadratic forms of given determinant $D$, which forms a group under composition, by an arbitrary abelian group $H$ whose elements are denoted by $\theta_{i}$. A correspondence is 
assumed between $H$ and the set $G$ of numbers $m>0$ which are prime to $M$ and of which $D$ is a quadratic residue. By considering the Dirichlet series $\sum a_{m}\left(\theta_{i}\right) \chi\left(\theta_{i}\right) \tau(m) m^{-s}$ summed over all $\theta_{i}$ in $H$ and all $m$ in $G$, where $a_{m}\left(\theta_{i}\right)$ is an integer determined by the correspondence, $\chi\left(\theta_{i}\right)$ is a group character for $H$, and $\tau(m)$ is a character modulo $m$, a result is obtained which includes Meyer's theorem as a special case, but is more general. (Received July 16, 1947.)

373. M. F. Smiley: The radical of an alternative ring. Preliminary report.

N. Jacobson's definition of the radical of an associative ring (Amer. J. Math. vol. $67(1945)$ pp. 300-320) is applied to alternative rings. It is shown that the resulting set is an ideal and certain elementary properties of the radical are established. This generalizes a result of M. Zorn (Ann. of Math. (2) vol. 42 (1941) pp. 676-686) concerning hypercomplex alternative rings, as well as a result of Dubisch and Perlis (Bull. Amer. Math. Soc. vol. 50 (1944) p. 53) concerning alternative algebras of finite order. A typical lemma encountered in the proof is that if $a+b+a b=0$ in an alternative ring $A$, then $a(b c)=(a b) c$ for every $c \in A$. (Received June 16, 1947.)

\section{4t. R. R. Stoll: Coset systems in semigroups. Preliminary report.}

Let $S$ denote a semigroup ( $=$ a system closed with repect to an associative binary operation). A partition $P=P\left(S_{\alpha}\right)$ of $S$ is called a left (right) coset system for $S$ if $x S_{\alpha}\left(S_{\alpha} x\right)$ is contained in a $P$-block $S_{\beta}$ for every $x . P$ is normal if it is both a left and right coset system and moreover is permutable with every coset system in $S$. The last condition means: for every coset system $Q$ the cover of a $Q$-block by $P$-blocks is a sum of $Q$ blocks. Analogues of the group theoretic results on composition and normal series are obtained. A theory of monomial representations is developed which generalizes those obtained by Rees (Proc. Cambridge Philos. Soc. vol. 36 (1940) pp. 387-400) for completely simple semigroups. (Received July 30,1947.)

\section{L. R. Wilcox: Closure operations. Preliminary report.}

Let $M$ be a set and $U(M)$ the set of all subsets of $M$. A semi-closure operation of $U(M)$ is an order homomorphism between $U(M)$ and itself such that $f(S) \supset S$ for $S \in U(M)$. If $f(f(S))=f(S)$, then $f$ is a closure operation. The present paper obtains formulas for the join and intersection of any set of closure operations and studies the composition $S \rightarrow f(g(S))$ of two closure operations. The composition, usually only a semi-closure operation, determines uniquely a closure operation by means of a transfinite sequence of extensions. Application is made to the case $M=A \times A$ ( $A$ a set); the elements $R \in U(M)$ are then binary relations on $A \times A$. Closure operations carrying any $R$ into its reflexive, symmetric and transitive extensions, respectively, are defined and found to possess many interrelations. Any relation $R$ gives rise to a unique closure operation in $U(A)$. It is proved that for such an operation the closed (that is, invariant) sets constitute a complete ring of sets; conversely, any complete ring arises in this manner from a unique reflexive and transitive $R$. (Received July 28, 1947.)

376. Margaret F. Willerding: Determination of all classes of positive quaternary quadratic forms which represent all (positive) integers.

It was apparently known to Diophantus and first proved by Lagrange (Oeuvres, vol. III, 1869, pp. 189-201) that the form $x^{2}+y^{2}+z^{2}+u^{2}$ represents all positive 
integers. Ramanujan (Proc. Cambridge Philos. Soc. vol. 19 (1916) pp. 11-12) proved that there are only 54 sets of positive integers $a, b, c, d$, such that $F=a x^{2}+b y^{2}+c z^{2}$ $+d u^{2}$ represents all positive integers. Dickson (Amer. J. Math. vol. 49 (1927) pp. 3956) called all such forms universal. Since these are reduced forms the result may be stated: the number of classes of forms which are equivalent to a form without cross products is equal to 54. It is of interest to ask what is the number of universal classes of forms if cross product terms are allowed. If the cross product terms have odd coefficients the number is quite large. If only the so-called "classic" forms, that is, forms whose cross product terms have necessarily even coefficients, are considered the number is not large. Employing an extension of the method of Ramanujan the theorem is proved: There are 178 classes of universal classic positive quaternary quadratic forms. (Received July 16, 1947.)

\section{ANALYsis}

\section{R. P. Agnew: Divergent series evaluable by matrix methods.}

Let $y_{n}=\sum_{k=1}^{\infty} a_{n k} x_{k}$ be a multiplicative matrix transformation with multiplier $\rho$; a sequence $x_{n}$ is evaluable $A$ to $L$ if $y_{n} \rightarrow L$ as $n \rightarrow \infty$. It is shown that if $\rho=0$, then $A$ evaluates each sequence $x_{n}$ (bounded or unbounded) which (1) oscillates sufficiently slowly and (2) is dominated by a sequence of positive numbers which becomes infinite sufficiently slowly. If $\rho \neq 0$ and $x_{1}, x_{2}, \cdots$ is a bounded divergent sequence evaluable $A$ to $L$, then the sequence $\left(x_{n}-L / \rho\right) \xi_{n}$ is evaluable $A$ whenever the sequence $\xi_{n}$ satisfies the conditions (1) and (2). (Received July 24, 1947.)

\section{Miriam C. Ayer and Tibor Rado: $A$ note on convergence in length.}

Let $\mathfrak{x}_{n}(t), t \in I, n=0,12, \cdots$, represent a sequence of vector functions with components $x_{n}(t), y_{n}(t), z_{n}(t)$ which are defined, continuous, and of bounded variation on the interval $I: a \leqq t \leqq b$, and which converge uniformly on $I$ to $x_{0}(t), y_{0}(t), z_{0}(t)$ respectively. Let $L\left(\mathfrak{x}_{n}\right)$ denote the length of the curve determined by the equations $x=x_{n}(t), y=y_{n}(t), z=z_{n}(t), a \leqq t \leqq b, n=0,1,2, \cdots$. Under these hypotheses the sequence $L\left(\mathfrak{x}_{n}\right)$ may or may not converge-the general situation being that $\lim \inf L\left(\mathfrak{x}_{n}\right) \geqq L\left(\mathfrak{x}_{0}\right)$. It is also well known that if $L\left(\mathfrak{x}_{n}\right) \rightarrow L\left(\mathfrak{x}_{0}\right)$ then the total variation of $c_{1} x_{n}(t)+c_{2} y_{n}(t)+c_{3} z_{n}(t)$ on $I$ converges to the total variation of $c_{1} x_{0}(t)$ $+c_{2} y_{0}(t)+c_{3} z_{0}(t)$ on $I$ for every choice of the (real) constants $c_{1}, c_{2}, c_{3}$. It is the purpose of this note to prove that conversely the convergence of the total variation of $c_{1} x_{n}(t)+c_{2} y_{n}(t)+c_{3} z_{n}(t)$ on $I$ to the total variation of $c_{1} x_{0}(t)+c_{2} y_{0}(t)+c_{3} z_{0}(t)$ on $I$ for every choice of the constants $c_{1}, c_{2}, c_{3}$ implies convergence of $L\left(\mathfrak{x}_{n}\right)$ to $L\left(\mathfrak{x}_{0}\right)$. This is a generalization of a result of A. P. Morse. (Received July 29, 1947.)

\section{9t. R. P. Boas: Simple sets of polynomials. Preliminary report.}

A simple set of polynomials $p_{n}(z)$ is one in which $p_{n}(z)$ is of degree $n$; we may write $p_{n}(z)=z^{n}+\sum_{k-0}^{n-1} p_{n} z^{k}$. The idea of the present investigation is that $p_{n}(z)$ is "nearly" $z^{n}$ if $|z|$ is large enough. The comparison theorem of Paley and Wiener (Fourier transforms in the complex domain, Amer. Math. Soc. Colloquium Publications, vol. 19, New York, 1934, p. 100) then leads to the following general result. If there is a sequence of constants $\beta_{n}$ such that $\left|p_{n k}\right| \leqq \beta_{n-k}$, the basic series (in the terminology of J. M. Whittaker) in terms of $p_{n}(z)$ represents in $|z|<r$ every function analytic in $|z|<r$ provided that $\sum_{k=1}^{\infty} \beta_{k} r^{-k}<1$; moreover, the expansion is unique. 
Several known theorems are special cases. The following corollaries appear to be new. If $\lim \sup \beta_{k}^{1 / k}<\infty$, the basic series represents every function analytic in a sufficiently large circle. If $p_{n}(z)$ is an Appell set $\left(p_{n}^{\prime}(z)=n p_{n-1}(z)\right)$, the basic series represents all entire functions of order 1 , type less than $r$, if $\sum_{n=1}^{\infty} p_{n 0} r-n / n !<1$. (Received July 22, 1947.)

380t. H. B. Curry and I. J. Schoenberg: On spline distributions and their limits: the Pólya distribution functions.

Define the truncated power function $x_{+}^{k-1}$ as $x^{k-1}$ if $x \geqq 0$, and as 0 if $x<0$ $(k=1,2, \cdots)$. Let $M_{k}(x ; z)=k(z-x)_{+}^{k-1}, \omega(x)=\left(x-x_{0}\right)\left(x-x_{1}\right) \cdots\left(x-x_{k}\right), x_{0}<x_{1}$ $<\cdots<x_{k}$. The divided difference of order $k$ of $M_{k}(x ; z)$, with respect to the variable $z$ for the values $z=x_{0}, x_{1}, \cdots, x_{k}$, that is (in Steffensen's notation) the function $M_{k}(x)=M_{k}\left(x ; x_{0}, x_{1}, \cdots, x_{k}\right)=\sum_{\nu=0}^{k} k\left(x_{\nu}-x\right)_{+}^{k-1} / \omega^{\prime}\left(x_{\nu}\right)$, is called a spline frequency function of order $k$. Its integral $\int_{-\infty}^{x} M_{k}(x) d x$ is called a spline distribution function. These functions have a number of interesting elementary properties of which the most noteworthy is the following identity concerning the divided difference of order $k$ of a function $f(x): f\left(x_{0}, x_{1}, \cdots, x_{k}\right)=(1 / k !) \int_{-\infty}^{\infty} M_{k}\left(x ; x_{0}, \cdots, x_{k}\right) f^{(k)}(x) d x$. The main result is as follows: Let $M_{k}(x)$ be a spline frequency function and assume that for a sequence of values of $k \rightarrow \infty, \lim \int_{-\infty}^{x} M_{k}(x) d x=L(x)$ as $k \rightarrow \infty$, in all continuity points of the distribution function $L(x)$. Then $L(x)$ is a Polya distribution function (see Proc. Nat. Acad. Sci. U.S.A. vol. 33 (1947) pp. 11-17), that is, its moment generating function $F(z)=\int_{-\infty}^{x} \exp (x z) d L(x)$ is the reciprocal of an entire function of the type $1 / F(z)=e^{-\gamma z^{2}+\delta z} \prod_{v=1}^{\infty}\left(1+\delta_{\nu} z\right) e^{-\delta_{\nu} z}\left(\gamma \geqq 0 ; \delta, \delta_{\nu}\right.$ real $)$. Conversely, every P6lya d.f. is the limit of a sequence of spline distribution functions. (Received July 28, 1947.)

\section{W. F. Eberlein and George Piranian: Ergodicity under semi- groups of Toeplitz transformations.}

The authors study the spectral analysis of Abelian semi-groups of regular Toeplitz transformations. The transformations are considered as mappings of the Banach space $m / c_{0}$ of equivalence classes of bounded sequences into itself (two sequences $x=x_{n}$ and $y=y_{n}$ are defined to be equivalent provided the sequence $x-y=x_{n}-y_{n}$ converges to zero). Special results are obtained for the semi-group generated by the Hölder transformation and for other semi-groups of Hausdorff transformations. For example, the set of points that are invariant under a given semi-group $G$ (that is, the set of fixed points with respect to $G$ ) is examined, and it is shown that the equivalence class of every sequence that oscillates sufficiently slowly is invariant under all regular Hausdorff transformations; in particular, the e.c. of the sequence $x_{n}=\sin \log \log n$ is invariant under the semi-group generated by the Hölder transformation, and the e.c. of the sequence $x_{n}=\sin \log \log \log n$ is invariant under the semi-group of regular Hausdorff transformations. A study is also made of the sets of points $X$ in $m / c_{0}$ that are ergodic with respect to a given Abelian semi-group $G$, that is, for which some sequence of transforms $B_{i} X=\sum_{j=0}^{i} a_{i j} A_{i j} X\left(i=0,1, \cdots ; \sum_{j=0}^{i} a_{i j}=1, a_{i j} \geqq 0, A_{i j}\right.$ in $G$ ) converges to a fixed point $Y$. (Received July 28,1947 .)

\section{2t. Marianne Freundlich: On normed rings.}

A completely continuous element of a normed ring $R$ is a ring-element which is completely continuous considered as a linear operator on the underlying Banach space. It is shown that if the ring is irreducible then completely continuous elements are in 
the radical. Weakly complete continuity is defined similarly and the set of weakly completely continuous elements is shown to be a closed ideal. If the radical of $R$ is the zero-ideal, then ring multiplication is continuous in both factors simultaneously and in each factor separately with no restrictions on $R$. Weakly complete continuity of every element is shown to be equivalent with reflexivity of $R$ if $R$ has a unit element, but the theorem may fail to hold in the absence of a unit. (Received July 28, 1947.)

\section{R. E. Fullerton: Representations of linear operators.}

The operators considered are bounded linear operators from a Banach space $X$ with range in a space of functions summable over a $\sigma$-field. The expression for representing an operator from a general Banach space to an $L$ space is given together with an expression for its norm. From this theorem representation theorems for operators from various function spaces to an $L$ space are derived. It is shown in particular that an operator from the space $L^{p}$ to the space $L$ has the form $T x=d / d s \int_{a}^{b} K(s, t) x(t) d t$ and its norm satisfies the inequality $\sup _{s}\left[\int_{a}^{b}|K(s, t)| p^{\prime} d t\right]^{1 / p^{\prime}}$ $\leqq\|T\| \leqq 2 \sup _{s}\left[\int_{a}^{b}|K(s, t)| p^{\prime} d t\right]^{1 / p^{\prime}}$ where $1 / p^{\prime}+1 / p=1$. If $T$ is a non-negative operator the equality holds between the first two members of the above expression. Various other representation theorems are given including new proofs of some already known. (Received July 28, 1947.)

384. R. E. Graves: An alternative definitition of an integral over the Wiener space. Preliminary report.

Let $C$ be the space of all continuous functions $x(t)$ on $[0,1]$ which vanish at $t=0$, and let $\left\{\alpha_{k}(t)\right\}(k=1,2, \cdots)$ be a complete orthonormal set on $[0,1]$, each of whose elements is of bounded variation. An $n$-dimensional $\alpha$-quasi-interval $Q$ is defined to be the set of all $x(\cdot) \in C$ such that $\xi_{i}<\int_{0}^{1} \alpha_{i}(t) d x(t) \leqq \eta_{i}(i=1,2, \cdots, n)$, and the area of $Q$ is defined as $\pi^{-n / 2} \int \cdots \int \exp \left[-\sum_{1}^{n} u_{j}^{2}\right] d u_{1} \cdots d u_{n}$, where the range of integration is $\xi_{i} \leqq u_{i} \leqq \eta_{i}(i=1,2, \cdots, n)$. The author shows that it is possible to construct a measure theory from this definition of the area of an $\alpha$-quasi-interval. An integral over $C$, termed the $\alpha$-integral, is defined on the basis of this measure theory. The author then proves that every $\alpha$-measurable functional is Wiener-measurable and that its $\alpha$-integral is equal to its Wiener integral. The paper concludes with a proof that, with certain restrictions on the set $\left\{\alpha_{k}(t)\right\}$, every Wiener-measurable functional is $\alpha$-measurable, and hence that the $\alpha$ and Wiener integrals are completely equivalent. (Received July 28,1947 .)

\section{P. R. Halmos: The range of a vector measure.}

This paper contains a simple proof of the fact that the range of a countably additive finite measure with values in a finite-dimensional real vector space is closed and, in the non-atomic case, convex. (Received July 9, 1947.)

\section{R. G. Helsel and E. J. Mickle: The Kolmogoroff principle for} the Lebesgue area.

Let $T: \mathfrak{r}=\mathfrak{r}(u, v)$ and $T^{*}: \mathfrak{r}=\mathfrak{r}^{*}(u, v)$, where $\mathfrak{x}$ is the vector with components $(x, y, z)$, be any two continuous transformations from the unit disk $D: u^{2}+v^{2} \leqq 1$ into $x y z$-space. If the inequality $\mid \mathfrak{x}\left(u_{1}, v_{1}\right)-\mathfrak{x}\left(u_{2}, v_{2}|\geqq| \mathfrak{x}^{*}\left(u_{1}, v_{1}\right)-\mathfrak{x}^{*}\left(u_{2}, v_{2}\right) \mid\right.$ is satisfied for every pair of points $\left(u_{1}, v_{1}\right),\left(u_{2}, v_{2}\right)$ in $D$, then the Lebesgue areas of the Fréchet surfaces determined by $T$ and $T^{*}$ satisfy the inequality $A(T) \geqq A\left(T^{*}\right)$. This result 
may be interpreted as showing that the Lebesgue area of a Fréchet surface satisfies the Kolmogoroff principle. (Received May 1, 1947.)

\section{Fritz Herzog: On upper and lower relative frequencies.}

Let $\left\{a_{k}\right\}$ be an infinite sequence of positive integers. Let $P(m ; n)$ denote the relative frequency with which the positive integer $m$ occurs among the first $n$ of the $a_{k}$. Then the numbers $H(m)=\lim \sup _{n \rightarrow \infty} P(m ; n)$ and $h(m)=\lim \inf _{n \rightarrow \infty} P(m ; n)$ are, respectively, the upper and lower relative frequencies of $m$ in the sequence. If the $a_{k}$ are allowed to assume all positive integers (infinite case) then the $H(m)$ and $h(m)$ satisfy the relations $0 \leqq h(m) \leqq H(m) \leqq 1$ and $H(m)-h(m)+\sum_{r=1}^{\infty} h(r) \leqq 1$ for all positive integral $m$. It is shown moreover that these relations are the only restrictions placed upon the $H(m)$ and $h(m)$. That means that to any given set of values for $H(m)$ and $h(m)$, satisfying the above conditions, there exists a sequence $\left\{a_{k}\right\}$, having the prescribed values of $H(m)$ and $h(m)$ as its upper and lower frequencies. If the $a_{k}$ are restricted to the integers $1,2, \cdots, M$ (finite case) then the most general relations (in the same sense as above) between the $H(m)$ and $h(m)$ are: $0 \leqq h(m) \leqq H(m) \leqq 1$, $H(m)-h(m)+\sum_{r=1}^{M} h(r) \leqq 1$ and $h(m)-H(m)+\sum_{r=1}^{M} H(r) \geqq 1$ for $m=1,2, \cdots, M$. (Received July $25,1947$.

388. W. H. McEwen: Derived series associated with an integrodifferential system.

Consider the integro-differential system $M(u)+\lambda u=0, U_{j}(u)=0(j=1,2, \cdots, n)$ in which $M(u) \equiv u^{(n)}+p_{1} u^{(n+1)}+\cdots+p_{n} u-\int_{a}^{b} h(x, \xi) u(\xi) d \xi+\sum_{1}^{n} \phi_{j}(x) l_{j}(u), \quad U_{j}(u)$ $\equiv W_{j}(u)-\int_{a} \psi_{j}(\xi) u(\xi) d \xi$, and $l_{j}(u), W_{j}(u)$ are linearly independent two-point boundary forms. Let $S_{N}(x)$ and $\sigma_{N}(x)$ denote the partial sums of the series expansions of the functions $f(x)$ and $M^{q}(f)$ relative to this system, $q$ being a given positive integer. If $f(x)$ satisfies $U_{j}(f)=0, U_{j}(M(f))=0, \cdots, U_{j}\left(M^{q-1}(f)\right)=0(j=1,2, \cdots, n)$ it is shown that $\left|f^{(k)}-S_{N}^{(k)}\right| \leqq K \int_{a}^{b}\left|M^{q}(f)-\sigma_{N}\right| d x$ for $k=0,1, \cdots, q n-1$; if in addition $M^{q}(f)$ is of bounded variation on $(a, b)$ these quantities converge uniformly to zero on $(a, b)$. (Received June 30, 1947.)

\section{G. R. MacLane: Approximation by finite Blaschke products.}

Let $R$ be the set of all non-constant functions $g(z)$ holomorphic in $|z| \leqq 1$ such that $|g(t)|=1$ for $|t|=1$. $R$ consists of all products of a finite number of linear transformations leaving the interior of the unit circle invariant. Let $E$ be the set of all functions $f(z)$ holomorphic in $|z|<1$ with $|f(z)| \leqq 1$. Theorem: $R$ is dense in $E$; that is, g:ven $f(z) \in E$, there exists $g_{n}(z) \in R(n=1,2, \cdots)$ such that $g_{n}(z) \rightarrow f(z)$ uniformly on any closed subset of $|z|<1$. Three different proofs are given: (1) starting with the Riemann image of $|z|<1$ by $w=f(z)$, a sequence of Riemann configurations corresponding to functions $g_{n}$ of $R$ is constructed so that $g_{n} \rightarrow f$. (2) Factoring out a Blaschke product and taking logarithms the desired approximation follows from the definition of an integral since the kernel of the Poisson integral is readily obtained as a special limit. (3) A third proof follows from the fact that there exists a function of $R$ of degree $n$ which assumes the same values as $f(z)$ at any $n$ given points. (Received July 14 , 1947.)

\section{Morris Marden: A refinement of Pellet's theorem.}

The principal theorem proved, applied and generalized in this paper is the following. Let $G\left(r_{0}, r ; p, \alpha\right)$ be the boundary curve of the gear shaped region obtained 
on deleting from the circle $|z| \leqq r$ the points common to the annular ring $r_{0}<|z|<r$ and the $p$ angles $(k=0,1,2, \cdots, p-1)\left(2 \alpha_{0}-\pi+4 \pi k\right) / 2 p \leqq \arg z \leqq\left(2 \alpha_{0}+\pi+4 \pi k\right) / 2 p$. Let $f(z)=a_{0}+a_{1} z+\cdots+a_{p} z^{p}+\cdots+a_{n} z^{n}, a_{p} \neq 0$, be a polynomial such that the real polynomial $F_{p}(z)=\left|a_{0}\right|+\left|a_{1}\right| z+\cdots+\left|a_{p-1}\right| z^{p-1}-\left|a_{p}\right| z^{p}+\left|a_{p+1}\right| z^{p+1}+\cdots$ $+\left|a_{n}\right| z^{n}$ has two positive zeros $r$ and $R$ with $r<R$. Then the real polynomial $\Phi_{p}(z)=\left[F_{p}(z)-a_{0}\right] / z$ has two positive zeros $r_{0}$ and $R_{0}$ with $r_{0}<r<R<R_{0}$ and the polynomial $f(z)$ has $p$ zeros in or on the curve $G\left(r_{0}, r ; p, \alpha_{0}\right)$ where $\alpha_{0}=\arg a_{0} / a_{p}$ and has no zeros between the curves $G\left(r_{0}, r ; p, \alpha_{0}\right)$ and $G\left(R, R_{0} ; p, \alpha_{0}\right)$. When $p=n$, this theorem coincides with the result due to Lipka in Monatshefte für Mathematik und Physik vol. 50 (1944) pp. 209-221. (Received July 16, 1947.)

\section{H. J. Miser: Generalized conformal representations of Fréchet surfaces.}

The author defines in terms of elementary properties an essentially nondegenerate transformation $T(P)=P^{*}$, where $T$ is continuous, $P$ is a 2-cell, and $P^{*}$ is a subset of Euclidean 3-space. If one of two Fréchet equivalent transformations is essentially nondegenerate, the other is also. Let $K$ be the class of Fréchet surfaces whose representations are essentially nondegenerate. Then saddle-surfaces and Lebesgue monotone surfaces (cf. McShane, Trans. Amer. Math. Soc. vol. 35 (1933) pp. 716-733) and nondegenerate surfaces (cf. Morrey, Amer. J. Math. vol. 57 (1935) pp. 692-702) are members of the class $K$. If a Fréchet surface $S$ is of class $K$ and has finite Lebesgue area, then $S$ admits of a generalized conformal representation; this theorem includes similar theorems of McShane (loc. cit.) and Morrey (loc. cit.). No "middle-space topology" is used in establishing the above results, while Morrey's proof that his theorem includes McShane's uses middle-space topology. Finally, using middle-space topology the author shows that the class $K$ is the class of nondegenerate surfaces, so that the new theorem stated here is equivalent to Morrey's theorem. (Received July 28, 1947.)

\section{C. N. Moore: Generalized limits in general analysis. IV.}

The present paper constitutes an extension to multiple limits of a theorem in general analysis dealing with simple limits, presented at a previous meeting (Bull. Amer. Math. Soc. Abstract 53-5-220). It deals with necessary and sufficient conditions that a certain functional operation $J$, which includes as special cases sequence to function transformations and function to function transformations by means of infinite integrals, shall be a limit-preserving transformation. (Received July 28, 1947.)

\section{G. B. Price: Researches in the theory of functions of several real} variables. I. Functions, limits, continuity.

The functions considered are general transformations $F^{(m)}: y_{j}=f_{j}\left(x_{1}, \cdots, x_{n}\right)$, $j=1, \cdots, m$. The $k+1$ points $X_{0}^{(n)}, X_{1}^{(n)}, \cdots, X_{k}^{(n)}, k \geqq 1$, determine an increment $\Delta\left[X^{(n)} ; X_{0}^{(n)} X_{1}^{(n)} \cdots X_{k}^{(n)}\right]$ of the independent variable $X^{(n)}:\left(x_{1}, \cdots, x_{n}\right)$. The images $F^{(m)}\left(X_{i}^{(n)}\right), i=0,1, \cdots, k$, of these points determine the corresponding increment $\Delta\left[F^{(m)} ; X_{0}^{(n)} X_{1}^{(n)} \cdots X_{k}^{(n)}\right]$ of the function $F^{(m)}$. The values of $\Delta\left[X^{(n)} ; X_{0}^{(n)} X_{1}^{(n)}\right.$ $\left.\cdots X_{k}^{(n)}\right]$ and $\Delta\left[F^{(m)} ; X_{0}^{(n)} X_{1}^{(n)} \cdots X_{k}^{(n)}\right]$ are the $k$-measures of the $k$-cells determined by the points $X_{i}^{(n)}$ and $F^{(m)}\left(X_{i}^{(n)}\right), i=0,1, \cdots, k$, respectively. The limits considered are limits of expressions such as $\Delta\left[F^{(m)} ; X_{0}^{(n)} X_{1}^{(n)} \cdots X_{k}^{(n)}\right]$ as $X_{1}^{(n)}, \cdots, X_{k}^{(n)}$ tend to $X_{0}^{(n)}$. If the increment of $F^{(m)}$ tends to zero as $X_{1}^{(n)}, \cdots, X_{k}^{(n)}$ tend to $X_{0}^{(n)}$ subject to certain specified conditions, then $F^{(m)}$ has the corresponding type of $k$ dimensional continuity. The results depend in an essential manner on the restrictions 
imposed on $X_{1}^{(n)}, \cdots, X_{k}^{(n)}$ as they tend to $X_{0}^{(n)}$. The case $k=1$ yields the ordinary theory of continuity. This paper contains an investigation of the various types of continuity and of their interrelations. The theory of determinants provides an essential tool. (Received July 24, 1947.)

\section{W. T. Reid: Expansion methods for the isoperimetric problem of Bolza in non-parametric form.}

In this paper sufficient conditions for a strong relative minimum in the isoperimetric problem of Bolza in non-parametric form are established by suitable modifications of the expansion method of proof previously used by the author (Ann. of Math. vol. 38 (1937) pp. 662-678; Trans. Amer. Math. Soc. vol. 42 (1937) pp. 183-190) for ordinary problems of Bolza in the calculus of variations. The present method of proof avoids the use of a Lindeberg theorem, in contrast to an earlier proof by the author (Duke Math. J. vol. 5 (1939) pp. 675-691) of a sufficiency theorem that is equivalent to the one herein given. (Received July 29, 1947.)

\section{Arthur Sard: Integral representations of remainders.}

Suppose that $X, Y, Z$ are Banach spaces; that $U$ is a linear operation on $X$ to all of $Y$; and that $V$ is a linear operation on $X$ to $Z$. If $V x=0$ whenever $U x=0, x \in X$, there is a linear operation $T$ on $Y$ to $Z$ such that $V x=T U x, x \in X$. This theorem and known representations of linear functionals on particular function spaces lead to explicit direct procedures for obtaining integral representations of the remainder in many processes of computation. Particular examples are cited. Among the theorems proved is the following. Let $C_{m}$ be the space of functions $x=x(s)$ with continuous $m$ th derivative on the finite interval $a \leqq s \leqq b$, norm, addition, and scalar multiplication being defined in the customary way. If the functional $V x$ is linear on $C_{n-p}, 0 \leqq p \leqq n$, and vanishes whenever $x$ is a polynomial of degree $n$, then $V x=\int_{a}^{b} x^{(n+1)}(s) \beta(s) d s$ for any function $x$ with absolutely continous $n$th derivative, where $\beta$ is a $p$-fold integral of a function of bounded variation and $\beta\left(s^{\prime}\right)=-V \phi_{s^{\prime}}, \phi_{s^{\prime}}=\phi_{s^{\prime}}(s)=\left(s-s^{\prime}\right)^{n} / n !$ if $s \leqq s^{\prime}$, $\phi_{s^{\prime}}(s)=0$ if $s>s^{\prime}$. The present paper is related to work of E. J. Rémès $(1939,1940)$. (Received July 28, 1947.)

\section{W: T. Scott: The corresponding continued fraction of a J-frac- tion.}

The author shows that there is a one-to-one correspondence between $J$-fractions, $K_{1}^{\infty}\left[c_{p} /\left(z+d_{p}\right)\right]$, and corresponding type continued fractions ( $C$-fractions) of the form $-K_{1}^{\infty}\left(-1 / b_{p} z^{\beta_{p}}\right)$, where $\beta_{1}=1, \beta_{p}$ is 0 or $1, \beta_{p}+\beta_{p+1}>0, b_{p} \neq 0$. The methods used for $J$-fractions are applicable to these $C$-fractions, and a theory parallel to that for $J$-fractions is obtained. This includes a theorem of invariability and a positive definite case related to those of Hellinger and Wall (Ann. of Math. vol. 44 (1943) pp. 103127), and a treatment of the indeterminate case similar to that of Wall (Bull. Amer. Math. Soc. vol. 52 (1946) pp. 671-679). (Received July 17, 1947.)

\section{F. C. Smith: An alternative form for some theorems of Ford and Newsom.}

In this paper, the author obtains a new form for the asymptotic expansion of a class of functions previously studied by W. B. Ford in his Asymptotic developments of 
functions defined by Maclaurin series (University of Michigan Press, 1936), chap. 4. The results can be generalized to give a theorem parallel to that proved by C. V. Newsom (Amer. J. Math. vol. 40 (1938) pp. 561-572). As in Ford's and Newsom's work, the proofs are based upon the use of contour integrals and the calculus of residues. (Received July 24, 1947.)

\section{Walter Strodt: Note on linear difference equations. Preliminary report.}

Given (1) $\sum_{j=0}^{n} A, y\left(x+\omega_{j}\right)=\phi(x)$, with $\phi(x)$ of type $(M, \gamma)$. (That is, $\phi(x)$ is analytic at $x=0$ and in the sector $S:|\arg x|<\gamma$, and for every positive pair $\epsilon, \delta$ there is a positive $C(\epsilon, \delta)$ such that $|\phi(x)|<C(\epsilon, \delta) \exp ((M+\epsilon)|x|)$ whenever $|\arg x|<\gamma-\delta$.) Given that $\omega_{0}=0$ and that $\omega_{1}, \cdots, \omega_{n}$ are in $\mathcal{S}$. Let $H(z)=1 /\left[\sum_{j=0}^{n} A_{j} \exp \left(\omega_{j} z\right)\right]$. Let $B$ be any point such that $H(B)$ is finite. Corresponding to $M$, but independently of $\phi(x)$, a constant $g$ and finitely many functions $I_{m}(t)(m=0,1, \cdots, s)$ are, by contour integration, constructed from the principal parts of $H(z)$, half-lines $\mathcal{L}_{1}, \mathcal{L}_{2}, \cdots, \mathcal{L}_{\text {s }}$ lying in $\mathcal{S}$ are determined, and finitely many poles $p_{1}, \cdots, p_{r}$ of $H(z)$ (orders $\left.j_{1}, \cdots, j_{r}\right)$ are selected, such that the most general solution of (1) of type $(M, \gamma)$ is $g \phi(x)+\int_{0}^{x} \phi(t) I_{0}(x-t) d t+\sum_{m=1}^{:} \int\left[B \phi(x+t)-\phi^{\prime}(x+t)\right] I_{m}(t) d t+D(x)$, where the second integral is taken over $\mathcal{L}_{m}$ and $D(x)$ is a linear combination, with arbitrary constant coefficients, of $x^{i} \exp \left(p_{k} x\right)\left(k=1, \cdots, r ; j=0,1, \cdots, j_{k}-1\right)$. The basic method is a generalization of the author's method of approximating q-difference equations (Ann. of Math. (1943); Amer. J. Math. (1947)). (Received July 29, 1947.)

399. Gerhard Tintner: Restricted maxima and minima which yield solutions homogeneous in some parameters.

There are $m+1$ functions $f, g^{(j)}(j=1, \cdots, m)$ of $n$ variables $x_{1} \cdots x_{n}$ and of $(N-n)$ parameters $x_{n+1} \cdots x_{N}$. By introducing $m$ Lagrange multipliers $\lambda_{j}$, form the function $F=f+\sum_{j} \lambda_{j} g^{(j)}$. Derivatives are denoted by subscripts. Necessary conditions for an extremum of $f$ with $g^{(j)}=0$ are: $F_{r}=0(r=1, \cdots, n)$. Sufficient conditions involve a set of restricted Hessians. They have the sign $\left(-1^{m}\right)$ for a minimum, are alternatively positive and negative for a maximum. A criterion for the solution $x_{r}$ to be homogeneous of zero degree in the parameters is the determinant $E_{r}=0 . E_{r}$ is formed by substituting into the $r$ th column of the last restricted Hessian the column: $-\left\{\sum_{k} x_{k} F_{l k} \cdots \sum_{k} x_{k} F_{n k} \sum_{k} x_{k} g_{k}^{(1)} \cdots \sum_{k} x_{k} g_{k}^{(m)}\right\}(k=n+1, \cdots, N)$. Important applications of homogeneous solutions occur in the modern theory of general equilibrium and employment in mathematical economics. (Received May 15, 1947.)

\section{Leonard Tornheim: On n-parameter families of functions and associated convex functions.}

An $n$-parameter family is a set of single-valued, real, continuous functions $f(x)$ on the interval $a \leqq x \leqq b$ such that for every set of points $\left(x_{i}, y_{i}\right)(i=1, \cdots, n)$ and $a \leqq x_{1}<x_{2}<\cdots<x_{n} \leqq b$ there is exactly one $f(x)$ with $f\left(x_{i}\right)=y_{i}$. An associated convex function is a function that intersects no member of the family more than $n$ times. Beckenbach and Bing have discussed 2-parameter families and T. Popoviciu has investigated the case of linear systems. Among the results obtained is the following: if $2 n$ sequences $\left(x_{i 1}\right),\left(y_{i 1}\right) ; \cdots ;\left(x_{i n}\right),\left(y_{i n}\right)(i=1,2, \cdots)$ converge to $x_{1}, y_{1} ; \cdots ; x_{n}$, $y_{u}$ respectively and if $x_{i j} \neq x_{i k}$ and, $x_{i k} \neq x_{i j}(j \neq k)$ then the sequence of functions 
$f_{i}(x)$ of the $n$-parameter family determined by $\left(x_{i j}, y_{i j}\right)(j=1, \cdots, n)$ converges uniformly to the function determined by $\left(x_{1}, y_{1}\right), \cdots,\left(x_{n}, y_{n}\right)$. (Received July 29, 1947.)

401. W. J. Trjitzinsky: Singular elliptic-parabolic partial differential equations.

This is a continuation of the author's extensive investigation of singular elliptic and hyperbolic partial differential equations, currently being published in Rec. Math. (Mat. Sbornik). In the present paper he is concerned chiefly with singular equations of elliptic-parabolic type; these are transformed (throughout the domain of definition) into integral equations in appearance of the first and second kind. (Received May 12, 1947.)

\section{2t. S. M. Ulam: On quasi-fixed points for transformations in func- tion spaces.}

A transformation $T$ of a topological space $E$ into itself has a quasi-fixed pointwith respect to a system of $k$ real-valued functions $f_{1}(p), \cdots, f_{k}(p)$ defined on $E$-if there exists a point $p_{0}$ such that $f_{1}\left(p_{0}\right)=f_{1}\left(T\left(p_{0}\right)\right) ; f_{2}\left(p_{0}\right)=f_{2}\left(T\left(p_{0}\right)\right) ; \cdots ; f_{k}\left(p_{0}\right)$ $=f_{k}\left(T\left(p_{0}\right)\right)$. This notion (of coincidence in the sense of Lefschetz between the transformation $L$ given by $f_{1}(p) \cdots f_{k}(p)$ and $L(T)$ is of interest in the case where $E$ is a function space- $T$ a (fixed point free) operator and $f_{1}, \cdots, f_{k} k$ given "properties" of the function which is an element of $E$, for example the first $k$ coefficients in a series development, the first $k$ moments of the function, and so on. Results are established on the existence of quasi-fixed points in the above sense for various special transformations-and systems of functionals occurring in analysis. The geometric properties of the manifold of all the quasi-fixed points in $E$ are investigated in the case where $T$ is an algebraic operator. (Received July 28, 1947.)

403. S. M. Ulam and John von Neumann: On combination of stochastic and deterministic processes. Preliminary report.

A computational procedure for the study of various differential equationsordinary or partial-is investigated. It consists of a statistical model of the corresponding physical problem and involves a process which is a combination of deterministic and stochastic processes (see Bull. Amer. Math. Soc. Abstract 51-9-165). This procedure is analogous to the playing of a series of "solitaire" card games and is performed on a computing machine. It requires, among others, the use of "random" numbers with a given distribution. Various distributions of such numbers can, however, be obtained by deterministic processes. For example, starting with almost every $x_{1}$ (in the sense of Lebesgue measure) and iterating the function $f(x)=4 x \cdot(1-x)$ one obtains a sequence of numbers on $(0,1)$ with a computable algebraic distribution. By playing suitable games with numbers "drawn" in this fashion, one can obtain various other distributions, either given explicitly or satisfying given differential or integral equations. (Received September 3, 1947.)

\section{Applied Mathematics}

404t. Stefan Bergman: A representation for the generating function of an operator in the theory of a compressible fluid.

The generating function $\mathrm{E}^{*}$ of the integral operator of the second kind $\mathrm{P}_{2}(f)=\int_{t=-1}^{1} H \mathrm{E}^{*} f\left[\mathrm{Z}\left(1-t^{2}\right) / 2\right] d t /\left(1-t^{2}\right)^{1 / 2}$ (see formulas [55] and [69]; here and in 
the following, numbers in brackets refer to NACA, TN 972), which operator generates streamfunctions $\psi(\lambda, \theta)=\operatorname{Im}\left[\mathrm{P}_{2}(f)\right]$ of compressible fluid flows, can be represented in the form $\mathrm{E}^{*}=\sum_{\kappa=1}^{2} A_{\kappa} \sum_{n=0}^{\infty} q^{(n, \kappa)} /\left(-t^{2} \mathrm{Z}\right)^{n-1 / 2+(2 / 3) \kappa}$, where $A_{\kappa}$ are constants, $\mathrm{Z}=\lambda+i \theta$; $\lambda=\lambda(M)$, and $\theta$ are cartesian coordinates of the "pseudo-logarithmic" plane (see [48]). The functions $q^{(n, \kappa)}=\sum_{\nu=0}^{\infty} C_{\nu}^{(n, \kappa)}(-\lambda)^{n-1 / 2+(2 / 3)(\kappa+\nu)}$ are connected by the equations $2(n-1 / 2+2 \kappa / 3) q_{\lambda}^{(n, \kappa)}+q_{\lambda \lambda}^{(n+1, \kappa)}+4 F q^{(n+1, \kappa)}=0, n=1,2, \cdots ; \kappa=1,2 ; q_{\lambda}^{(n)}$ $=d q^{(n)} / d \lambda, \cdots$, where $4 F=\lambda^{-2} S, S=(5 / 36)+\sum_{\nu=2}^{\infty} \alpha_{\nu}(-\lambda)^{2 \nu / 3}$, is given by [71]. $\mathrm{E}^{*}$ satisfies [88] (with $F_{m}$ replaced by $F$ ), and $\left|C_{\nu}^{(n, \kappa)}\right|^{2} \leqq C 2^{n} / s_{0}^{\nu}$, where $s_{0}$ is the radius of convergence of $S(s), s=(-\lambda)^{2 / 3}, C$ being a suitably chosen constant. Continuing the solutions $\psi(\lambda, \theta)$ to complex values of the first argument, assuming $\Lambda=0$, in $\psi(\lambda+i \Lambda, \theta)$ in the subsonic case, and $\lambda=0$ in the supersonic case, the author obtains "mixed" flow patterns. (Received September 1, 1947.)

\section{5t. Stefan Bergman: Two-dimensional mixed flow patterns.}

In this paper the coefficient $l(\mathrm{H})$ in the equation $S(\psi) \equiv l(\mathrm{H}) \psi_{\theta \theta}+\psi_{\mathrm{HH}}=0$ for the streamfunction of a compressible fluid is replaced by $-c_{1} \mathrm{H}, c_{1}$ being a constant (for notation see NACA, TN No. 972 , p. 25$)$. The new equation $S \dagger(\psi)=0$ admits a comparatively simple mathematical treatment. In particular the generating function $\mathbf{E} \dagger$ of the integral operator $\mathrm{P} \dagger(f) \equiv \int_{t=-1}^{1} \mathrm{E} \dagger(\lambda, \theta, t) f\left[2^{-1}(\lambda \dagger+i \theta)\left(1-t^{2}\right)\right] d t /\left(1-t^{2}\right)^{1 / 2}$ of the second kind which generates solutions of $S \dagger(\psi)=0$ (see the above TN, p. 28) can be represented in the form $\mathbf{E} \dagger=c_{2}(-2 \lambda \dagger)^{-1 / 6} F(1 / 6,5 / 6,1 / 2, \chi)+c_{3}(-2 \lambda \dagger)^{-2 / 3}$ $\cdot\left[-t^{2}(\lambda \dagger+i \theta)\right]^{1 / 2} F(2 / 3,4 / 3,3 / 2, \chi)=c_{4}\left[-t^{2}(\lambda \dagger+i \theta)^{-1 / 6} F(1 / 6,2 / 3,1 / 3,1 / \chi)\right.$ $+c_{5}(-2 \lambda \dagger)^{2 / 3}\left[-t^{2}(\lambda \dagger+i \theta)\right]^{5 / 6} F(5 / 6,4 / 3,5 / 6,1 / \chi)$ where $x=\left[t^{2}(\lambda \dagger-i \theta) / 2 \lambda \dagger\right]$, $-\lambda \dagger=c_{6}(-\mathrm{H})^{3 / 2}, F$ the hypergeometric series, $c_{k}$ are constants. Analogous formulas hold for the supersonic region, in which case $\lambda \dagger$ is replaced by $\Lambda=i \lambda \dagger, \Lambda=c_{7} \mathrm{H}^{3 / 2}$. Sufficient conditions for $f$ are given in order that the streamfunction $\psi=\operatorname{Im}[\operatorname{P} \dagger(\psi)]$ defined in the subsonic may be continued to the supersonic region. It appears that the results can be extended to the case of the exact equation $S(\psi)=0$. (Received August 18, 1947.)

\section{H. E. Goheen: A non-rigorous suggestion for numerical solu-} tion of certain problems in heat generation.

The partial differential equation (1) $\partial T / \partial t=\partial^{2} T / \partial x^{2}+(2 / x)(\partial T / \partial x)+e^{-1 / T}$ with boundary.conditions (a) at $t=0, T=\theta_{0}, 0<x<a$, and $T=\theta_{1}, x>a$; (b) at $x=\infty$, $T=w$ in which $w$ is the solution of the ordinary differential equation $w^{\prime}=e^{-1 / w}$ with $w(0)=\theta_{1}$, and the partial differential equation (2) $\partial u / \partial t=\partial^{2} u / \partial x^{2}+e^{-1 / u}$ with boundary conditions (a) at $t=0, T=\theta_{0}$ if $|x|<a$ and $u=\theta_{1}$ if $|x|>a$; (b) at $|x|=\infty$, $u=w$, as above, are discussed. Assuming $T(x, t)$ in (1) and $u(x, t)$ in (2) to possess derivatives of all orders with respect to $x$ at $x=0$, a technique is developed which reduces the determination of each of these functions and their derivatives w.r.t. $x$ at $x=0$ to an infinite set of ordinary differential equations. Making a second assumption that the solution of each of these sets is approximated sufficiently well by a finite number (two or three) of them, an integration technique is determined. Making a third assumption that $T(x, t)$ and $u(x, t)$ are analytic functions of $x$ at $x=0$ with infinite radius of convergence, these values of derivatives and functions may be used to obtain the values of $T(x, t)$ and $u(x, t)$ for any $x$ and $t$. (Received June 13,1947.)

407t. F. G. Gravalos: A consequence of Karman's law of similarity. A complete logical exposition of Karman's law of similarity of turbulent flow is 
lacking. Based on Karman's own remarks, an attempt is made to fill that gap. As a consequence of such analysis, the phenomenology of turbulent boundary layers may be given a new formulation; namely that, "The boundary layer's Reynolds number is a functional of the local velocity profile." (Received July 24, 1947.)

\section{A. E. Heins: Water waves over a channel of infinite depth with a dock.}

The author is concerned with the solution of $\left(^{*}\right) \Delta \phi(x, y)-k^{2} \phi(x, y)=0$ in the halfspace $-\infty<x<\infty, y \geqq 0$ subject to the boundary conditions (a) $\partial \phi / \partial y=0$ at $y=0$, $x<0$ and $\partial \phi / \partial y=\beta \phi$ at $y=0, x>0(\beta>0)$. The velocity potential $\phi(x, y)$ is assumed to possess a logarithmic singularity at $x=0$ in order that $\left(^{*}\right)$ have a traveling wave term for $x \gg 0$. It is further assumed that $\phi(x, y)=O\left(e^{\gamma_{r}}\right), r=\left(x^{2}+y^{2}\right)^{1 / 2}$ for $r \rightarrow \infty$ and $\gamma<k$. The surface $y=0, x<0$ is the dock-like surface, while $y=0, x>0$ is the free surface of the liquid. This boundary value problem may be formulated as an integral equation of the Wiener-Hopf type in terms of a Green's function kernel which may be exhibited explicitly. The Fourier transform (in the complex domain) is used to solve this integral equation. It is found that there exists a traveling surface wave on the surface $x>0, y=0$ provided $\beta^{2}>k^{2}$. Fourier methods are not applicable to the case $k=0$. It is possible to provide the asymptotic form of $\phi(x, y)$ for $x, y \gg 0$ as well as $x, y \rightarrow 0$. The technique of solution of the Wiener-Hopf integral equation has been recently applied by $\mathrm{H}$. Feshbach and the present writer to a related boundary value problem in acoustics. (Received July 16, 1947.)

409. M. Z. Krzywoblocki: On the two-dimensional steady turbulent flow of a compressible fluid far behind a solid symmetrical body (momentum transfer theory).

Schlichting solved the equations of a steady turbulent flow of an incompressible fluid in a wake far behind a solid symmetrical body by application of the method of successive approximations. In the present paper this method is extended to apply to the solution of the equations of a steady turbulent flow of a compressible fluid in a wake far behind a solid symmetrical body. As is known, the complete tensor form for the eddy stress is due to Prandtl. Two other complete tensor forms for the stresses due to density fluctuations are proposed by the author. Throughout all the calculations in the paper the monentum transfer theory was used. This was done purposely. In the theory of incompressible turbulent flow it is the opinion that the velocity distributions by the momentum transfer theory or the vorticity transfer theory are the same. The question arises whether it is true for a compressible turbulent flow. To answer this question, the author intends to solve the entire system of equations based on both theories. In the present paper he solves the first part. The second part will be treated in a separate paper. (Received July 11, 1947.)

\section{C. G. Maple and J. L. Synge: Aerodynamic symmetry of proj- ectiles.}

According to the usual hypothesis, the aerodynamic force system (force plus couple) on a projectile is a function of its instantaneous motion (velocity plus angular velocity). If the projectile possesses symmetry, the dependence of the force system on the instantaneous motion is conditioned by this symmetry. In this work, a projectile with $n$-gonal symmetry is considered, with or without reflectional symmetry rela- 
tive to a plane containing the axis of $n$-gonal symmetry, and it is assumed that the elements of the force system can be expanded in power series in the components of cross-velocity and cross-spin, the coefficients depending on the axial components of velocity and spin. The effect of symmetry is to make some of these coefficients disappear, and to establish odd or even dependence on axial spin in the case of surviving coefficients. If axial and cross-spin vanish, expansion for the axial torque starts with the $n$th power of the cross-velocity. (Received July 28, 1947.)

411. P. F. Neményi and R. C. Prim: On the equations of plane rotational flow of a perfect gas in natural coordinates and on plane rotational gas flow with constant velocity magnitude or constant vorticity along the streamlines.

The partial differential equations governing steady plane rotational flow of a perfect gas are referred to a natural coordinate system consisting of the streamlines and an orthogonal family of curves. These equations are used to investigate the possibility of flows in which the velocity is constant along each streamline. It is proved that these two types of flow are possible only in streamline patterns consisting of concentric circles or of parallel lines. (Received July 30,1947.)

412. P. F. Neményi and R. C. Prim: Some patterns of vorticose flow of a perfect gas.

Complete analytic solutions of definite boundary value problems of compressible, rotational flows of a perfect gas are extremely difficult to obtain. Therefore, in the present stage of study of rotational gas flow the inverse, or semi-inverse, approach promises to give most instructive solutions. In the present paper broad families of flow patterns satisfying the differential equations of steady, rotational gas flow and having throughout the field certain prescribed geometric or kinematic properties are studied. Beltrami and Massotti have investigated classes of incompressible flow patterns characterized by certain specific relations between the velocity and vorticity vector fields. In the present paper the implications of the same kinematic conditions are investigated for the case of compressible flow. In addition, an exhaustive survey is made of all steady flows of a perfect gas for which, in a cylindrical, isometric coordinate system, the three velocity components depend only on one coordinate. It is proved that the only generating coordinate net for such solutions consists of logarithmic spirals and their limiting cases. (Received July 30,1947.)

413t. John von Neumann and H. H. Goldstine: Some estimates on the numerical stability of the elimination method for inverting matrices of high order.

Since computing machines give necessarily only approximately correct products and quotients, it is important, especially for high speed devices, to determine the loss of precision caused by given numerical procedures in their application to specific problems. The well known elimination method for inverting high order matrices is particularly important since it involves highly iterative as well as implicit algorithms. In the present paper it is shown that if the matrix at hand is definite, a slightly modified version of this method yields an inverse in error below $20 n^{2} \epsilon / \mu^{2}$ where $n$ is the order of the matrix, $\epsilon$ the roundoff error, and where $\mu$ is the smallest proper value 
of the matrix, if the latter is so adjusted that all its elements are below $1 / 2$. It is observed that the method depends on the decomposition of the matrix in question into a product $C^{*} C$, where $C$ is semidiagonal. The proposed algorithm produces a matrix $C_{1}$ for which $C_{1}{ }^{*} C_{1}$ lies very close to the correct $C^{*} C$. This estimate proves to be the really relevant one, and not that one of the deviation of $C_{1}$ from $C$. Extension to indefinite matrices is also discussed. (Received August 21, 1947.)

\section{Mary H. Payne and W. T. Payne: Geometrical spinor theory.}

The bipolar coordinates of a line element on the light cone are defined and their transformation properties stated. The transformation properties are the same as for a first rank spinor, so that the bipolar coordinates of a line element on the light cone may be regarded as the components of a first rank spinor. The scalar and spinor products of two first rank spinors are defined and interpreted geometrically. The direct product is shown to lead to a second rank spinor, and certain geometrical properties of second rank spinors are discussed. (Received July 23, 1947.)

\section{George Pólya: Torsional rigidity increases by symmetrization.}

For example, a semicircle with radius $a$ is changed by symmetrization with respect to its bounding diameter into an ellipse with semiaxes $a$ and $a / 2$. The torsional rigidity for the former cross-section is $.296 a^{4} G$, for the latter $.314 a^{4} G$. The general theorem is closely analogous to the following: The pitch of the gravest tone of a membrane decreases by symmetrization. In fact, both theorems can be proved by the same method, given for the membrane by G. P6lya and G. Szegö, Amer. J. Math. vol. 67 (1945) p. 14. Incomparably simpler is the proof for the following theorem: The polar moment of inertia of a plane area with respect to its centroid decreases by symmetrization. (Received July $25,1947$.

416. L. V. Robinson: The solution of linear partial differential equations by differential operator methods. Preliminary report.

By making use of generalized differential operators, the formulas which give the solution of an ordinary linear differential equation can be extended to solve partial differential equations of the type $\partial u / \partial x+P(x, y, z) \partial u / \partial y+Q(x, y, z) \partial u / \partial z+R(x, y, z) u$ $=S(x, y, z)$. These operators are of the type exp $\left[G_{1}(x, y, z) \partial / \partial y+G_{2}(x, y, z) \partial / \partial z\right]$; and a table is given for the various forms of the functions, $G$. The work involved in solving such equations is thereby considerably reduced. (Received July 28, 1947.)

\section{A. E. Schild: Discrete space-time and integral Lorentz trans- formations.}

A cubic lattice of points in Minkowski space-time is considered, the four coordinates of the lattice points being integers. The "integral" Lorentz transformations, which leave the cubic lattice invariant as a whole, form an infinite, discrete six-parameter group. In a certain sense the cubic lattice is homogeneous and spatially isotropic. It may therefore be suitable as a background for physical theory. (Received July 3, 1947.)

418. A. C. Sugar: The coding of boundary value problems for an electronic computer. Preliminary report.

First, using the Liebmann form of the finite difference method for finding an ap- 
proximate solution of the Dirichlet problem (which is actually a specialization of the Gauss-Seidel iterative process), a code is prepared for carrying out this process on an electronic digital computer. A generalization (by abridgment) of the UNIVAC instruction Code C-5 was used. First the rectangular boundary was considered and then the non-rectangular boundary. It is planned to consider the biharmonic equation for rectangular and non-rectangular boundaries and finally to code the more general Gauss-Seidel process and by comparison determine the extent of the advantages derived from the specialized codes. (Received September 3, 1947.)

\section{C. A. Truesdell: On the reliability of the membrane theory of shells of revolution.}

The author investigates the correctness of the stress and moment resultants predicted by the membrane theory of shells of revolution when the load and support are not rotationally symmetric and when the boundary conditions are consistent with a state of membrane stress. These distributions are consistent with the results of ordinary three-dimensional elasticity when the apex of the shell is open or when it is closed but flat, but he shows that they may be unrealistically sensitive to slight changes in the meridian curve when the apex is pointed. The method of proof throughout depends upon the stress functions of Némenyi and the author's prior investigation of the membrane theory (Trans. Amer. Math. Soc. vol. 58 (1945) pp. 96-166). The author suggests a possible substitute theory based on stress and moment averages rather than stress and moment resultants, and not requiring that the shell radius be much smaller than its thickness. This theory if properly constructed agrees with the present membrane theory when that theory is correct, but deviates from it in problems involving pointed shells. The equilibrium equations of this theory are easy to establish, but it is not clear what the proper approximate relations giving the stress and moment averages in terms of the displacements should be. (Received June 30, 1947.)

\section{C. A. Truesdell and R. Schwartz: On the Newtonian mechanics of continua.}

The author states the principles governing the motion of all Newtonian continua and gives formulations of the theories of perfectly elastic bodies and of viscous, compressible, heat conducting fluids. The analysis is valid for inhomogeneous, nonisotropic media. To the familiar principles of conservation of mass and momentum must be added the principle of conservation of energy stated sufficiently generally to be valid in the motion of inhomogeneous media. To this end a local phenomenological theory of the thermodynamics of inhomogeneous media is constructed. Murnaghan's theory of finite deformations of an elastic solid is generalized so as to apply to nonhomogeneous bodies non-uniformly heated. A viscous fluid is defined as a continuum in which the rate of dissipation of energy per unit volume is a function only of the thermodynamic state, the mean stress, and the deformation rate tensor. This definition leads to a nonlinear theory of viscous fluids, to which the Navier-Stokes theory is a first order approximation. (Received July 30,1947.)

\section{Henry Wallman: Electronic general-transform computer.}

A proposed method is described for rapid electronic evaluation of general integral transforms of the form $F(x)=\int_{a}^{b} f(t) K(x, t) d t$, where $f(t)$ is an arbitrary real function 
and $K(x, t)$ an arbitrary real kernel. The complete computation consumes about .01 second and is repetitively presented on the face of a cathode-ray tube. The instrument consists of (a) a high-speed one-variable function table for $f(t)$, scanned in $100 \mu$ sec, (b) a high-speed two-variable function table for $K(x, t)$, scanned in two dimensions, as in television, in $10,000 \mu \mathrm{sec}$, (c) an electronic multiplier, (d) an electronic averager, (e) a synchronized cathode-ray tube system. Easily treated special cases include the sine and cosine transform and the convolution integral. Because of its speed and the ease of varying the one-variable function table, the instrument promises to be capable of solving integral equations of various types, and, as a special case, of inverting matrices. (Received August 3, 1947.)

\section{Alexander Weinstein: On the torsion of shafts.}

The author investigates the torsion of a family of shafts with axial semi-infinite holes and with flat bottoms. The corresponding profiles are obtained by the method of sources and sinks in a five-dimensional space, the sources being distributed on a fourdimensional disk of variable radius and of a constant total strength. The investigation is based on previous results by the author on many-valued stream functions in generalized potential theory (Bull. Amer. Math. Soc. vol. 53 (1947) pp. 59, 494). (Received July 15, 1947.)

\section{GeOMEtry}

\section{P. O. Bell: Projective curvature and projective torsion of a curve on an analytic surface.}

The author presents new geometric characterizations for Bompiani's projective curvature and the author's projective torsion of a curve $C_{\lambda}$ on an analytic surface $S$ at a point $P_{x}$. Let $t_{\lambda}$ be the tangent to $C_{\lambda}$ at $P_{x} ; l^{\prime}$ a line through $P_{x}$ intersecting the quadric of Lie in two points $P_{x}$ and $Q$; and $K$ the cone, with vertex at $P_{x}$, which contains the conic of intersection of the tangent plane to the quadric of Lie at $Q$ with the quadric of Wilczynski at $P_{x}$. To characterize projective curvature let $l^{\prime}$ be the projective normal to $S$ at $P_{x}$, and let $\pi_{n}, \pi, \pi_{0}, \pi_{ \pm}$be respectively the plane determined by $t_{\lambda}$ and $l^{\prime}$, the tangent plane to $S$ at $P_{x}$, the osculating plane of $C_{\lambda}$ at $P_{x}$ and the planes through $t_{\lambda}$ which are tangent to the cone $K$, respectively. The projective curvature is given by the cross-ratio equations $x= \pm\left(\pi_{n}, \pi, \pi_{0}, \pm \pi\right)$. To characterize the projective torsion $\tau$ of $C_{\lambda}$ at $P_{x}$ replace in these cross-ratio equations $k$ by $2 \tau$, the plane $\pi_{n}$ by $\pi_{0}$, and the planes $\pi_{0}, \pi_{ \pm}$by the planes $p_{3}, p_{ \pm}$, respectively, defined as follows: $p_{3}$ is the plane determined by $t_{\lambda}$ and the point $d^{3} x / d s^{3}$ where $d s$ is the Fubini element of projective arc length, and $p_{ \pm}$are the planes through $t_{\lambda}$ tangent to the cone $K$ defined for the line $l^{\prime}$ which joins the points $P_{x}$ and $d^{2} x / d s^{2}$. The covariant points $d^{2} x / d s^{2}$, $d^{3} x / d s^{3}$ have been geometrically characterized by the author (Trans. Amer. Math. Soc. vol. 50 (1941) p. 539). (Received July 25, 1947.)

\section{H. S. M. Coxeter: Coordinates in the projective plane.}

To define the homogeneous coordinates of a point $P$ for a given triangle of reference $X Y Z$ and unit point $U$ : on the conic $P U X Y Z$, take an arbitrary point $I$; set up a parameter (or "abscissa") on the conic, taking the values $0,1, \infty$ at the respective points $U, I, P$; let $x, y, z$ be the values of the parameter at $X, Y, Z$; then $x, y$, are the desired coordinates for $P$. They are homogeneous, since a different choice of $I$ would have the effect of multiplying them all by the same constant. Initially, $x, y, z$ 
must be all distinct and different from zero, but this restriction can be removed later. Coordinates in $n$ dimensions can be set up in an analogous manner, using a rational normal $n$-ic curve instead of a conic. (Received June 23,1947.)

425. J. M. Field: A kinematic characterization of series of lineal elements in the plane and of their differential invariants under the group of whirl-similitudes and some of its subgroups.

In a previous paper (Bull. Amer. Math. Soc. vol. 48 (1942)) the author investigated the geometry of lineal elements under the seven-parameter group of whirlsimilitudes $G_{7}$. Now he determines a set of fundamental differential invariants of series of lineal elements under $G_{7}$ and under two of its subgroups: $G_{6}$ (group of whirlmotions) and $G_{3}$ (group of whirls). It is shown that series and their differential invariants under these groups can be given simple kinematic characterizations. (Received March 31, 1947.)

\section{Michael Goldberg: Circular-arc rotors in regular polygons.}

A rotor is defined here as a convex closed curve which remains tangent to all the sides of a fixed polygon during a complete rotation of the curve. The curves of constant width (which may rotate within a pair of parallel fixed lines) are a special class of rotors. The conception of rotors in regular polygons, the proof of their existence, and a method of constructing them have been attributed to Kakeya by Fujiwara (Tôhoku Science Reports vol. 4 (1915) pp. 303-312). Among these rotors are curves composed entirely of arcs of circles, but such curves have been described previously only for the equilateral triangle, the square and the regular pentagon. The author gives a general construction for obtaining a circular-arc rotor for each regular polygon. It has been shown by Blaschke (Math. Ann. vol. 76 (1914) pp. 504-513) and Fujiwara (Proc. Imp. Acad. Tokyo vol. 3 (1927) pp. 307-309 and vol. 7 (1931) pp. 300-302) that the rotors of least area for the triangle and the square are circular-arc rotors. The other circular-arc rotors, described in this paper, may conceivably share this minima! property. (Received April 2, 1947.)

\section{V. G. Grove: Generalized canonical lines.}

A generalization of the class of canonical lines associated with a point on an analytic surface is presented in this paper. To this class of generalized canonical (g.c.) lines belong the classical canonical lines. A geometric method of generating these g.c. lines is made to depend on an invariant family of curves, and an invariant point on the tangent to that curve of the family through the generic point of the surface. In particular the invariant family of curves used in the generation of the classical canonical lines is any one of the family of Segre curves. Generalizations of the integral invariants used by Wilkins to generate the classical lines may be used to generate the g.c. lines. Generalizations of the scroll directrices of Sullivan follow from these integral invariants. (Received June 23, 1947.)

428. S. B. Jackson: Geodesic vertices on surfaces of constant curvature.

In a former paper by the writer (Bull. Amer. Math. Soc. vol. 50 (1944) pp. 564578) there were obtained five structural properties of plane curves with only two vertices. The present paper investigates the extent to which these results can be ex- 
tended to curves having only two extrema of the geodesic curvature and lying in a simply-connected region of a surface of constant curvature. Four of the properties are still valid while the fifth has to be modified. A new property of such curves is established, namely that such curves have only a finite number of double points and arcs. The methods parallel closely those of the earlier paper, and use is made of the former results by mapping into the plane. Two theorems are established, analogous to well known results in the plane, relating the number of geodesic vertices on a curve to its number of intersections with a geodesic circle. It is not necessary in any of the results to specify the curvature of the surface or even its sign. (Received July 15 , 1947.)

429t. W. H. Roever: A geometric construction for choosing in their proper relation the projective scales on the axonometric axes of perspective axonometry.

During the recent war much use was made of production illustration in the design of airplanes. The construction given by the author is applicable not only to the production of such illustration but also to the solution in the picture itself of the geometric problems of space from the data contained in the picture. (Received August 4,1947.)

\section{Statistics ANd Probability}

\section{L. A. Aroian and Marguerite D. Darkow: The fourth degree} exponential function.

It is shown that the fourth degree exponential function is supported by the Bernoulli probability function and the hypergeometric probability function as well as being the function for which the method of moments is the "best" method according to the criterion of maximum likelihood. In the general situation six moments, at most, are needed. The function is classified into two general groups depending on symmetry or asymmetry and each case is divided again into unimodal and bimodal distributions. Examples show that the function is very successful in graduating the main Pearson types and the Gram-Charlier Type A frequency function. Various generalizations of the exponential function are indicated. In addition to its wide generality, the greatest practical advantage of the new system is the simplicity of the numerical calculations. (Received July 23, 1947.)

\section{K. L. Chung: On the maximum partial sums of sequences of in- dependent random variables.}

The asymptotic behavior of the maximum partial sums of a sequence of independent random variables is studied in this paper. Two groups of new limit theorems are established under general conditions. The first group deals with theorems of the "weak" type. The limiting distribution of the maximum partial sums is obtained with an estimate of the remainder, thus improving a recent result of Erdös and Kac. Another estimate is obtained for a different domain of variation, which plays an essential role in the sequel. These results correspond to the sharper forms of the central limit theorem. In the second group theorems of the "strong" type are obtained, giving precise lower bounds (in the sense of probability) for the maximum partial sums. These results form the exact counterpart to the general form of the law of the iterated logarithm, due to Feller, which give the precise upper bounds. A summary of the 
main results and methods has appeared in Proc. Nat. Acad. Sci. U.S.A. vol. 33 (1947), pp. 132-136. (Received July 21, 1947.)

\section{Harold Hotelling: Effects of non-normality at high significance} levels.

The effects of non-normality in the underlying population on the probabilities of "significance" by customary statistical tests are not well understood, in spite of numerous attacks, both mathematical and experimental, on the problem. Chung's proof that the distribution of the Student ratio $t$ has in samples from an arbitrary population a distribution approaching normality for large samples tends to confirm the idea that non-normality makes little difference if only the sample is fair'y large. But this holds only for a fixed range of values of $t$ while the sample number $N$ increases. If $p$ is the probability that $|t|>t_{0}$ in samples of $N$ from a normal population and $p^{\prime}$ is the corresponding probability for another population, it is shown that $\lim _{N \rightarrow \infty}\left\{\lim _{t_{0} \rightarrow \infty}\left(p^{\prime} / p\right)\right\}$ may be zero or infinite or may take any finite value. Conditions that this limit be unity are concerned only with the "shoulders" of the population histogram, and have nothing to do with its moments or behavior at infinity. This suggests caution in applying familiar tests with high significnce levels and that the use of moments cannot lead to the most appropriate criterion of non-normality for this purpose. (Received July 16, 1947.)

\section{3t. P. L. Hsu: A general weak limit theorem for independent dis-} tributions.

For every positive integer $n$ let there be $n$ distribution functions $F_{n 1}(x), F_{n 2}(x)$, $\cdots, F_{n n}(x)$. Assume that $\lim _{n \rightarrow \infty} \max _{1 \leqq j \leqq n}\left\{1-F_{n j}(x)+F_{n j}(-x)\right\}=0$. Let $F(x)$ be the convolution $F_{n 1}(x)^{*} F_{n 2}(x)^{*} \ldots{ }^{*} F_{n n}(x)$. Let $\psi(t)=m i t+\int_{-\infty}^{+\infty}\left(e^{i t x}-1-i t x /\left(1+x^{2}\right)\right)$ - $\left(\left(1+x^{2}\right) / x^{2}\right) d G(x)$, with $G(x) \uparrow$ and $G(\infty)-G(-\infty)<\infty$. Let $F(x)$ be the (infinitely divisible) distribution law having $\exp \psi(t)$ as its characteristic function. In order to have $\lim _{n \rightarrow \infty} F_{n}(x)=F(x)$ at every continuity point of $F(x)$, it is necessary and sufficient that the following relations hold at every $x$ greater than 0 such that $\pm x$ are continuity points of $G(y)$ : (I) $\lim _{n \rightarrow \infty} \sum_{j=1}^{n} \int_{|y|>x} d F_{n j}(y)=\int_{|y|>x}\left(\left(1+y^{2}\right) / y^{2}\right) d G(y)$, (II) $\lim _{n \rightarrow \infty} \sum_{j=1}^{n}\left\{\int_{|y|<x} y^{2} d F_{n j}(y)-\left(\int_{|y|<x} y d F_{n j}(y)\right)^{2}\right\}=\int_{|y|<x}\left(1+y^{2}\right) d G(y), \quad$ (III) $\lim _{n \rightarrow \infty} \sum_{j-1}^{n} \int_{|y|<x} y d F_{n j}(y)=m+\int_{|y|<x} y d G(y)-\int_{|y|<x}(1 / y) d G(y)$. (Received June 1, 1947.)

\section{E. L. Lehmann and Henry Scheffé: On the problem of similar} regions.

If $X=\left(X_{1}, \cdots, X_{n}\right)$ is a set of random variables with a joint probabi ity density depending on a set of parameters $\theta=\left(\theta_{1}, \cdots \theta_{m}\right)$, and if $T=\left(T_{1}, \cdots, T_{m}\right)$ is a set of sufficient statistics for $\theta$, then Neyman (Philos. Trans. Roy. Soc. London Ser. A vol. 236 (1937) pp. 333-380) has proved that a region $w$ in the space of $X$ is similar with respect to $\theta$ (that is, has constant probability for all $\theta$ ) if it has the following structure: The intersections $w(t)$ of $w$ with the surfaces $T=t$ have the property that the conditional probability of the sample point $X$ falling into $w$ given that $T=t$ does not depend on $t$. In the present paper a necessary and sufficient condition is found for the regions with the above structure to be the only similar regions. This condition is shown to be satisfied for a certain class $K$ of probability densities which contains as special cases all densities for which the totality of similar regions has been previously determined. In particular the partial differential equations which Neyman (Ann. Math. Statist. 
vol. 12 (1941) pp. 46-76) assumed were satisfied in his solution of the problem of similar regions are solved and it is shown that any density satisfying these equations belongs to the above class $K$. (Received July 10, 1947.)

\section{Herman Rubin: Some results on the distribution of quadratic forms from Gaussian stochastic processes. Preliminary report.}

If one considers the estimation of the parameters of a Gaussian stochastic process whose elements are continuous functions from the functional values over a finite interval, one often finds that certain parameters can be estimated exactly, and certain parameters can not. This result of ten depends on the distribution of quadratic functionals whose arguments are elements of the stochastic process under consideration. In this paper, it is shown that the elements of a certain class of quadratic functionals have distributions concentrated at a point, and that the elements of a different class do not; in this latter case, the characteristic function is computed. (Received July 26, 1947.)

\section{D. F. Votaw: Testing compound symmetry in a normal multi- variate distribution.}

Let $X$ be a $t$-order vector variate $(t \geqq 3)$ having d.f. $F(X)$. Let the components of $X$ be divided into mutually exclusive subsets. $F(X)$ is said to be compound symmetric, for the given division of its variates into subsets, if it is invariant over all permutations of its variates within these subsets. $F(X)$ is completely symmetric if the invariance holds over all permutations of its variates. In a normal, compound symmetric $F(X)$ there is equality of means, of variances, and of covariances within each subset of variates and equality of covariances between any two subsets of variates. In this paper likelihood ratio criteria are developed for various hypotheses involving compound symmetry in a normal distribution and in $k$ normal distributions $(k \geqq 2)$. Given that the corresponding null hypothesis is true, the moments of each criterion are obtained explicitly and the distribution of each criterion is identified as the product of independent beta variates. For the one-sample case, the distributions are given explicitly for $t=3,4,5$ for certain divisions of the variates into subsets. In a previous paper Wilks gave a very thorough treatment of the case of complete symmetry. (Received July $21,1947$.

\section{J. E. Walsh: Some significance tests for the mean using the sample range and midrange.}

Consider a sample of size $n(2 \leqq n \leqq 10)$ drawn from a normal population with mean $\mu$. Let $x_{n}$ be the largest value and $x_{1}$ the smallest value of the sample. Significance tests are developed to compare $\mu$ with a given hypothetical value $\mu_{0}$ by use of the sample. These tests are based on the quantity $D=\left[\left(x_{1}+x_{n}\right) / 2-\mu_{0}\right] /\left(x_{n}-x_{1}\right)$ $=[$ (sample midrange $)-($ hypothetical mean $)] /$ (sample range). One-sided and symmetrical tests are considered. Values of $D_{\alpha}$ such that $\operatorname{Pr}\left(D>D_{\alpha} \mid \mu=\mu_{0}\right)=\alpha$ are computed for $\alpha=.05, .025, .01, .005$. These values of $D_{\alpha}$ can be used to obtain one-sided tests at the $.05, .025, .01, .005$ significance levels and symmetrical tests at the $.10, .05, .02, .01$ significance levels. Efficiencies are computed for one-sided tests at the .05 and .01 significance levels. The efficiency is at least 90 percent for $n \leqq 6$ at the .05 significance level and for $n \leqq 8$ at the .01 level. The range-midrange test can be applied without computation through the use of an easily constructed graph. The 
application of a test requires only the plotting of the sample point $\left(x_{1}, x_{n}\right)$ on this graph. (Received July 14, 1947.)

\section{TOPOLOGY}

438. Emil Artin and R. H. Fox: On the complementary domains of spheres and simple closed curves in 3-space.

In spherical 3-space examples are constructed of (a) a simply connected closed surface whose interior, though simply connected, is not homeomorphic to the interior of the unit sphere; (b) a simply connected closed surface each of whose complementary domains is homeomorphic to the interior of the unit sphere although no homeomorphism of space upon itself can transform the surface into the unit sphere; (c) a simple closed curve which bounds a 2-cell and whose complementary domain has an infinite cyclic fundamental group although no homeomorphism of space upon itself can transform the curve into the unit circle. (Received July $25,1947$.

439. Beno Eckmann, Hans Samelson and G. W. Whitehead: $A$ note on fiber bundles.

It is proved that it is impossible to fiber the $n$-sphere $S_{n}$ (the Euclidean $n$-space $E_{n}$ ) with the torus $T_{m}$ as fiber if $m>1$, for odd $n$, and if $m \geqq 1$, for even $n$. The proof makes use of the Eckmann-Hurewicz-Steenrod relations for homotopy groups in fiber spaces, and the Eilenberg-MacLane relations between homotopy and homology groups. (Received July 29, 1947.)

\section{Samuel Eilenberg: Homology groups of cartesian products.}

The cartesian product $K \times L$ is defined for abstract complexes $K, L$. If in each dimension the integral groups of chains of $K$ and $L$ are free abelian groups, then the integral homology group $H_{r}(K \times L)$ is isomorphic with the direct sum $H_{p}\left(K, H_{q}(L)\right)$, $p+q=r$, of the homology groups of $K$ with coefficients in the integral homology groups of $L$. Using universal coefficient theorems various other forms of the relation are obtained. (Received July 28, 1947.)

\section{O. H. Hamilton: Fixed points for interior transformations.}

It is shown that, if $T$ is an interior continuous transformation of a bounded locally connected plane continuum $M$ which does not separate the plane onto a topological 2-cell which contains $M$, then $T$ leaves a point of $M$ invariant. It is shown further that if $T$ is an interior continuous transformation of a topological 2-cell $I$ onto a continuum $M$ which contains $I$, then $T$ leaves a point of $I$ invariant. (Received August $6,1947$.

442. E. H. Larguier: Homology bases with applications to local connectedness.

The author studies sequences of direct-sum decompositions of the (augmented) homology group $H^{r}(S, \mathfrak{F})$ of a compact Hausdorff space $S$ with coefficients over a field $\mathfrak{F}$. These give rise to sequences of subspaces of $H^{r}(S, \mathfrak{F})$, called decomposing sequences, and also to associated sequences of $r$-cycles of $S$, which serve to determine bases for $H^{r}(S, \mathfrak{F})$, suitably topologized. The converse procedure from sequences of $r$-cycles to decomposing sequences and finally to direct-sum decompositions is also 
possible. Conditions are given under which $H^{r}(S, \mathfrak{F})$ becomes a metric space; and further restrictions are indicated which assure the completeness of $H^{r}(S, \mathfrak{F})$. The homology group of a compact $G_{\delta}$ subset of a locally compact $l c^{r}$ space verifies this abstract situation. It is further shown that if there exists a sequence of 0 -cycles which can be associated with a decomposing sequence of subspaces, then there also exists a sequence of nontrivial 0-cycles carried by pairs of points associated with the same decomposing sequence. The application of the preceding concepts leads to necessary and sufficient conditions for imbedding a class of compact spaces in "minimal" $0-l c$ spaces, and incidentally to an extension of certain results of Wilder (Fund. Math. vol. 19, pp. 4564) to non-metric compact spaces. (Received July 9, 1947.)

\section{Paul Olum: Homology with operators and mapping theory.}

Let $K$ be a finite connected simplicial $n$-complex and $T$ an arcwise connected and locally arcwise connected topological space. The author is principally concerned with the problem of determining the homotopy classes of mappings of $K$ into $T$. Let $\pi_{r}(T)$ denote the $r$ th homotopy group of $T$. Then the classification is known when $\pi_{r}(T)=0$ for $r>1$, and when $\pi_{r}(T)=0$ for $r<n$, as well as for some special cases. The author gives a simple and effective classification in the case $\pi_{r}(T)=0$ for $1<r<n$. The classification is given in terms of the following topological quantities associated with $K$ and $T$ : the fundamental group of $K, \pi_{1}(K)$; the groups $\pi_{1}(T)$ and $\pi_{n}(T)$; the operation of $\pi_{1}(T)$ on $\pi_{n}(T)$; a function $k_{n+1}$ which assigns to $n+1$ ordered elements of $\pi_{1}(T)$ an element of $\pi_{n}(T)$; and an $n$-dimensional cohomology group with operators in $K$. This last is substantially the same as Steenrod's cohomology with local coeffcients. There is also proved an extension theorem for the mappings of an $(n+1)$ complex into such a space $T$. This gives a necessary and sufficient condition that a mapping of the $n$-skeleton into $T$ can be extended over the whole complex. (Received July $25,1947$.

\section{E. H. Spanier: Cohomology theory for general spaces.}

Using a definition due to A. D. Wallace, a new cohomology theory for general spaces is defined in terms of functions from sets of points in the space to the coeffcient group. This definition is quite similar to some definitions previously given by J. W. Alexander (Proc. Nat. Acad. Sci. U.S.A. vol. 21 (1935) pp. 509-512 and Ann. of Math. vol. 37 (1936) pp. 698-708). It is shown that this theory satisfies the cohomology axioms of Eilenberg and Steenrod (Proc. Nat. Acad. Sci. U.S.A. vol. 31 (1945) pp. 117-120). The new groups coincide with the Cech cohomology groups on compact Hausdorff spaces and agree with the singular cohomology groups on locally finite polyhedra. Hence, the new theory is distinct from any of the theories now in use. (Received May 15, 1947.)

\section{5t. A. D. Wallace: Group-invariant continua.}

Let $X$ be a compact connected Hausdorff space, $Z$ a topological space which is also a group and $f$ a map of $Z \times X$ into $X$ such that $f(e, x)=x, f(z,(w, x))=f(z w, x)$ where $e$ is the identity in $Z$. Define $z(x)=f(z, x)$. The author is concerned with the proposition: There exists a subcontinuum $E$ of $X$ with no cutpoint such that $z(E)=E$ for all $z$ in $Z$. In this note the proposition is shown to be valid provided $Z$ is abelian or connected or finite. When $X$ is assumed metric and $Z$ cyclic the result was first proved by W. L. Ayres ( $X$ locally connected) and extended by J. L. Kelley. (Received June 4, 1947.) 
446. J. W. T. Youngs: Extensions of a homeomorphism defined on the boundary of a 2-manifold.

Given a 2-manifold, $M$, with boundary, $B$, and a homeomorphism, $h: B \rightarrow B$, this paper concerns the extension of $h$ to a homeomorphism $h^{*}: M \rightarrow M$. If $M$ is orientable an extension need not exist; there is a necessary and sufficient condition for the extendability of $h$. If $M$ is non-orientable, then $h$ can always be extended. (Received July $24,1947$.

T. R. HollCroft, Associate Secretary 